Review Article

\title{
Mathematical and Metaheuristic Applications in Design Optimization of Steel Frame Structures: An Extensive Review
}

\author{
Mehmet Polat Saka ${ }^{1}$ and Zong Woo Geem ${ }^{2}$ \\ ${ }^{1}$ Department of Civil Engineering, University of Bahrain, P.O. Box 32038, Isa Town, Bahrain \\ ${ }^{2}$ Department of Energy and Information Technology, Gachon University, Seongnam 461-701, Republic of Korea \\ Correspondence should be addressed to Zong Woo Geem; zwgeem@gmail.com
}

Received 28 September 2012; Accepted 4 December 2012

Academic Editor: Sheng-yong Chen

Copyright (c) 2013 M. P. Saka and Z. W. Geem. This is an open access article distributed under the Creative Commons Attribution License, which permits unrestricted use, distribution, and reproduction in any medium, provided the original work is properly cited.

\begin{abstract}
The type of mathematical modeling selected for the optimum design problems of steel skeletal frames affects the size and mathematical complexity of the programming problem obtained. Survey on the structural optimization literature reveals that there are basically two types of design optimization formulation. In the first type only cross sectional properties of frame members are taken as design variables. In such formulation when the values of design variables change during design cycles, it becomes necessary to analyze the structure and update the response of steel frame to the external loading. Structural analysis in this type is a complementary part of the design process. In the second type joint coordinates are also treated as design variables in addition to the cross sectional properties of members. Such formulation eliminates the necessity of carrying out structural analysis in every design cycle. The values of the joint displacements are determined by the optimization techniques in addition to cross sectional properties. The structural optimization literature contains structural design algorithms that make use of both type of formulation. In this study a review is carried out on mathematical and metaheuristic algorithms where the effect of the mathematical modeling on the efficiency of these algorithms is discussed.
\end{abstract}

\section{Introduction}

Structural analysis and structural design are two inseparable tools of a structural designer. Design process necessitates finding out the cross-sectional properties of the members of a steel frame such that the frame with these members has the required strength to withstand the external loadings, and its deflected shape is within the limitations specified by design codes. Structural designer can determine the cross-sectional properties of steel frame members in one step if the frame is statically determinate, if there are no restraints on joint displacements. In such frames computation of member forces does not require the prior information of cross-sectional properties, and the design can be completed within one step of structural analysis. Despite the design of statically indeterminate frames, structural analysis cannot be carried out without knowing the values of cross-sectional properties which makes the design process iterative. Designer has to first assume certain values for the cross-sectional properties or select certain steel profiles from the available list of steel sections for the frame members before the analysis of the frame can be carried out in order to find out internal forces and moments in the members. With these assumed or preselected values of member cross-sectional properties, the structural response of the frame may not be within the limitations imposed by the design codes or the response might be far away from the bounds which are an indication of an overdesign. In this case, the designer has to change the values of the originally adopted cross sectional properties in order to satisfy the conditions that the values of joint displacements and strength of its members are within the limitations imposed by design codes. It is not difficult to envisage that designer has to carry out several trials before the desired set of cross-sectional properties is obtained. Although some engineering experience and intuition can be used in assigning the cross-sectional properties of members, it is needless to mention that finding the best combination of these cross-sectional properties is quite time consuming and 
a complex task. It requires tremendous computational time to try all the possible combinations such that the strength and displacement constraints imposed by the design codes are satisfied, and the amount of steel which is required to build the frame is the minimum. However, the emergence of computational methods of mathematical programming and improvements that took place in computers in early 1960's has provided another approach for handling the structural design problems. In this new approach, the design problem is formulated as a decision-making problem where an objective function is to be minimized or maximized, while number of constraint functions is satisfied. The formulation of structural design problems as decision-making problems has yielded a new branch in structural engineering called structural optimization [1-12].

The mathematical model of a decision-making problem has the following form:

$$
\begin{array}{ll}
\text { Minimize } & W=f\left(x_{i}\right), \quad i=1, \ldots, n, \\
\text { Subject to } & h_{j}\left(x_{i}\right)=0, \quad j=1, \ldots, \text { ne, } \\
& g_{j}\left(x_{i}\right) \leq 0, \quad j=\mathrm{ne}+1, \ldots, m, \\
& x_{i}^{\ell} \leq x_{i} \leq x_{i}^{u},
\end{array}
$$

where $x_{i}$ represents decision variable $i$. Decision variables may take continuous or discrete values. Continuous decision variable can take any real value within the range shown in (1d). The decision variables in structural optimization are called design variables which are the parameters that control the geometry or material properties of a steel frame. For example, the moment of inertias of beams and columns in a frame can be considered as continuous design variable if these elements are to be manufactured locally for that particular frame. However, if they are to be selected from commercially available set of steel sections, design variables can only take isolated values which make them discrete variables.

$f\left(x_{i}\right)$ in (1a) represent the objective function which can be used as a measure of effectiveness of the decision. In structural optimization problems generally minimum weight or cost of the structure is taken as objective function. The equalities $h_{j}\left(x_{i}\right)$ and inequalities $g_{j}\left(x_{i}\right)$ in (1b) and (1c), respectively, represent the limitations imposed on the behavior of the structure by the design codes. These may be serviceability limitations or ultimate strength requirements if the design code considered is based on limit state design. However, if the design code is based on allowable stress design then the constraints given in (1b) and (1c) become the stiffness equalities, displacement, and/or allowable stress limitations.

The review of the mathematical formulation of design optimization of steel frames is carried out according to the historical developments that took place in the design methods. Earlier steel design codes were based on allowable stress design concept, while the later design codes use limit state design perception.

\section{Formulation of Steel Frame Design Optimization Problem}

Survey on the structural optimization literature reveals that there are basically two types of design optimization formulation for the optimum design of elastic steel skeletal framed structures although some variations of each type do exist. In the first type of formulation which is also called conventional formulation or coupled analysis and design (CAND) only cross-sectional properties of steel frame members are taken as design variables. Joint displacements are not treated as design variables. In such formulation the stiffness equations are excluded from the mathematical model. Consequently, when the values of cross-sectional properties change during design cycles, it becomes necessary to analyze the structure and update the joint displacements and members forces. Hence structural analysis is a complementary part of the design process. In the second type of formulation joint displacements are also treated as design variables in addition to the cross-sectional properties of frame members. In such formulation the stiffness equations are included as design constraints in addition to other limitations in the mathematical model. This eliminates the necessity of carrying out structural analysis in every design cycle. The values of the joint displacements are determined by the optimization techniques similar to those of cross-sectional properties. It is apparent that in this type of formulation the total number of design variables is much more than the first type of formulation. This type of formulation is called simultaneous analysis and design (SAND). It should be pointed out that both ways of formulation yield the same optimum result. However, depending on the type of formulation adopted in the design problem the size and mathematical complexity of the model acquired differ.

2.1. Coupled Analysis and Design (CAND). In this type of formulation design phase is separated from the analysis phase. This is achieved by only treating the cross-sectional properties such as areas or moment of inertias of members as design variables. The general outlook of the mathematical model of this type of formulation is given in the following if allowable stress design method is considered for the frame:

$$
\begin{array}{ll}
\text { Minimize } & W=\rho \sum_{i=1}^{\mathrm{ng}} \ell_{i} A_{i}, \quad i=1, \ldots, \mathrm{ng} \\
\text { Subject to } & \delta_{j}\left(A_{i}\right) \leq \delta_{j u}, \quad j=1, \ldots, \mathrm{rd} \\
& \sigma_{k}\left(A_{i}\right) \leq \sigma_{\mathrm{all}}, \quad k=1, \ldots, \mathrm{nm}, \\
& A_{i l} \leq A_{i} \leq A_{i u},
\end{array}
$$

where $\rho$ is the density of steel, $A_{i}$ represents cross-sectional area for group, and $i$ and $\ell_{i}$ is the total length of the members in group $i$ in the steel frame. $\delta_{j}\left(A_{i}\right)$ is the restricted $j$ th displacement, $\delta_{j u}$ is its upper bound, and $\mathrm{rd}$ is the total number of such restricted displacements. $\sigma_{k}\left(A_{i}\right)$ is the maximum stress in member $k$, and $\sigma_{\text {all }}$ is the allowable stress for steel. $\mathrm{nm}$ is the total number of members in the structure. 
TABLE 1: $a_{i}$ and $b_{i}$ values for $\mathrm{W}$ sections.

\begin{tabular}{lcc}
\hline$i$ & $a_{i}$ & $b_{i}$ \\
\hline 1 & 1.3162 & 2.1650 \\
2 & 0.5971 & 1.8220 \\
3 & 1.0160 & 1.5710 \\
\hline
\end{tabular}

In the previously mentioned model joint displacements and stresses that develop in a frame due to external loading are expressed as function of cross-sectional areas of members. It is apparent that determination of the response of the frame under the applied loads requires the values of not only areas of members groups but the other cross-sectional properties such as moment of inertia and sectional modulus. Therefore in order not to increase the number of design variables in the programming problem, it becomes necessary to relate the other cross-sectional properties to sectional areas. This is achieved by applying the least square approximation to sectional properties of practically available steel profiles. These relationships can be expressed in the following form:

$$
I_{x}=a_{1} A^{b_{1}}, \quad I_{y}=a_{2} A^{b_{2}}, \quad Z=a_{3} A^{b_{3}},
$$

where $A$ is the area, $I_{x}$ and $I_{y}$ are the moments of inertia about $x-x$ and $y-y$, axis and $Z$ is the sectional modulus of the cross-section. $a_{1}, a_{2}, a_{3}, b_{1}, b_{2}, b_{3}$ are constants. The values of these constants are obtained for the W sections of AISC [13] by making use of the least square approximation in [14]. The values of these constants are given in Table 1 . It should be noted that in their computation the unit of centimeter $(1 \mathrm{~cm}$ $=10 \mathrm{~mm}$ ) was used.

It is apparent from (2b) that restricted displacements are required to be computed to find out whether they satisfy the displacement limitations. The joint displacements in framed structures are related to external loads through stiffness equations; $[K]\{D\}=\{P\}$ where $[K]$ is the overall stiffness matrix, $\{D\}$ is the vector of joint displacements, and $\{P\}$ is the vector of joint loads. The joint displacements can be computed from the stiffness equations as $\{D\}=$ $[K]^{-1}\{P\}$. Although here the inverse of overall stiffness matrix is required to be calculated in order to obtain the joint displacements, this is avoided due to its high computational cost in the application of the optimization techniques used in the solution of the problem. Instead derivatives of the overall stiffness matrix with respect to design variables are calculated depending on the optimization technique selected.

2.2. Simultaneous Analysis and Design (SAND). In this type of formulation joint displacements are also treated as design variables in addition to cross-sectional properties. Since the cross-sectional properties and joint displacements are implicitly related through stiffness equations, it becomes necessary to include the stiffness equations as design constraints in the mathematical model. The general outlook of the mathematical model of such formulation is given in the following:

$$
\begin{array}{ll}
\text { Minimize } & W=\rho \sum_{i=1}^{\mathrm{ng}} \ell_{i} A_{i}, \quad i=1, \ldots, \mathrm{ng}, \\
\text { Subject to } & K\left(A_{i}\right)\{D\}=\{P\} \\
& \delta_{j} \leq \delta_{j u}, \quad j=1, \ldots, \mathrm{rd}, \\
& \sigma_{k}\left(A_{i}, \delta_{j}\right) \leq \sigma_{\mathrm{all}}, \quad k=1, \ldots, \mathrm{nm}, \\
& A_{i \ell} \leq A_{i} \leq A_{i u},
\end{array}
$$

where $\rho$ is the density of steel, $A_{i}$ represents the crosssectional area selected for group $i$, and $\ell_{i}$ is the total length of the members in group $i$ in the frame. ng is the total number of groups in the steel frame. $A_{i \ell}$ and $A_{i u}$ are the lower and upper bounds imposed on the cross-sectional area $i$. Equality constraint (4b) represents stiffness equations. $\{D\}$ is the vector of joint displacements, which are treated as design variables. $\delta_{j}$ is the restricted joint displacement, and $\delta_{j_{u}}$ is its bound. rd is the total number of restricted displacements. $\sigma_{k}\left(A_{i}, \delta_{j}\right)$ represents stress constraint which is a function of area and end joint displacements of member $k . \sigma_{\text {all }}$ is the allowable stress for steel. $\mathrm{nm}$ is the total number of the members in the structure.

This way of formulation of the design problem is an integrated formulation which combines design and analysis phases in the same step that eliminates the need of separate structural analysis in each design cycle. Furthermore in this formulation the displacement constraints becomes very simple. Because they are treated as design variables they turn out to be just upper bound constraints. However, the number of design variables and constraints become very large compared to the previous type of formulation which necessitates use of powerful optimization techniques that are efficient for largescale problems. It should be noticed that further variable transformation is necessary for joint displacement variables in this type of formulation, if mathematical programming techniques are used for the solution of the design problem due to the fact that while joint displacements can be positive or negative, mathematical programming techniques operate with only positive variables. This brings further mathematical burden to a designer. Furthermore, the difference in magnitude between the cross-sectional properties and joint displacements causes numerical difficulties in the solution of the design problem which makes it necessary to normalize the design variables.

Saka $[15,16]$ carried out reviews of the mathematical modeling and solution algorithms presented in recent years for the optimum design of skeletal structures. The review first introduces the general formulation of the general structural optimization problem based on linear elastic behavior without referencing to a particular design code. Later implementation of the design requirements defined in design codes is also covered. The techniques developed for the solution of optimum design problems are reviewed in a historical order. 
Arora and Wang [17] also presented the review of mathematical modeling for structural and mechanical system optimization. In their review it is stated that the formulations are classified into three broad categories. The first type of formulation is known as the conventional formulation where only structural design variables are treated as optimization variables. In the second type of formulation state variables such as node displacements and/or element stresses are treated as design variables in addition to the cross-sectional properties. This type of formulation is called as simultaneous analysis and design (SAND). The third type of formulation is known as a displacement-based two-phase approach where displacements are treated as unknowns in the outer loop and the cross-sectional properties are treated as the unknowns in the inner loop. The review also covers more general formulations that are applicable to economics, optimal control, multidisciplinary problems, and other engineering fields. Altogether 254 references are included in the review.

Optimum design problem of steel frames turns out to be a nonlinear programming problem if allowable stress design is adopted whichever formulation method explained previously is used. The numerical optimization techniques available in the literature for obtaining the solution of nonlinear programming problems can be broadly classified into two groups such as deterministic and stochastic algorithms. Deterministic optimization techniques make use of derivatives of the objective function and constraints in the search of the optimum solution and involve no randomness in the development of solution techniques. The other groups of techniques that are also called metaheuristic optimization techniques do not require the derivatives of the objective function and the constraints. They rely on random search paradigms based on simulation of natural phenomena. These methods are nontraditional search and optimization methods, and they are very suitable and efficient in finding the solution of combinatorial optimization problems.

\section{Deterministic Solution Techniques for Elastic Steel Frame Design Optimization Problems}

Historically mathematical programming techniques were first to be used to obtain the solution of optimum design problem [1-12]. The developments took place in computers and in the methods of mathematical programming, and they have initiated a new era in designing engineering structures. The need to design aircraft components to have minimum weight and later the requirements of space programs has provided the necessary funds and motivation to engineers to develop effective design tools. As a result of a large number of research works, numerous numerical techniques were developed for the solution of nonlinear programming problems [1-12]. However, when these techniques were applied to the design of real-size practical steel frames numerical difficulties were encountered. It is found out that mathematical programming-based structural optimization methods were only capable of designing steel frames with few dozen of design variables.

Optimality criteria approach was introduced in late 1960 with the purpose of overcoming the previously mentioned discrepancies of mathematical programming techniques. It was shown that the optimality criteria method was not dependent on the size of design problem and obtained the near-optimum solution with few structural analyses. It was this feature that made the optimality criteria techniques attractive over the mathematical programming methods. Optimality criteria approaches were widely utilized in the design of large-size practical structures.

One of the common features of the both methods is that they consider continuous design variables. In reality, variables in most of the steel frame design problems are to be selected from a set of discrete values that are available in practice. This fact made it necessary to extend the mathematical programming techniques to be capable of handling discrete programming problems. This necessity has brought further difficulties, and the capability of the methods developed to solve such programming problems was found to be limited. Arora [18] recently presented comprehensive review of the methods available for discrete variable structural optimization. In spite of the fact that the number of algorithms has been developed for obtaining the solution of discrete variables steel frame design optimization problems, they have not found widespread application owing to their complexity and inefficiency in dealing with large size structures.

3.1. Mathematical Programming Based Steel Frame Design Optimization Techniques. Mathematical programming techniques start the search for the optimum solution at a preselected initial point and compute the gradients of the objective function and constraints at this point. They take a step in the negative direction of the gradient of the objective function in the case of minimization problems to determine the next point. They continue this process of finding a new point until there is no significant change in the values of design variables within two consecutive iterations. There are several mathematical programming techniques available in the literature. In this paper only those that are used in developing structural optimization algorithms for the optimum design of steel frames are considered. These can be collected in three broad groups. Each group uses different concept. In the first group of algorithms approximation is carried out through the use of linearization concept. The second group converts the constrained problem into unconstrained one, and the third group handles the mathematical programming problem as it is without transforming it into another form.

3.1.1. Sequential Linear Programs. The first group of algorithms employs sequential linear programming method developed by Griffith and Stewart [19]. This method makes use of Taylor expansions to transfer the nonlinear programming problem into a linear programming one. This is achieved by taking first two terms after applying Taylor's expansion to nonlinear functions in the programming problem. The design problems (2a), (2b), (2c), (2d), (4a), (4b), 
(4c), (4d), and (4e) can be rewritten in a general form as in the following:

$$
\begin{array}{ll}
\text { Minimize } & W=\sum_{k=1}^{m} w_{k}\left(x_{i}\right), \quad i=1, \ldots, n, \\
\text { Subject to } & h_{j}\left(x_{i}\right)=0, \quad j=1, \ldots, \mathrm{ne}, \\
& g_{j}\left(x_{i}\right) \leq 0, \quad j=\mathrm{ne}+1, \ldots, \mathrm{nc}
\end{array}
$$

where $x_{i}$ represents the design variable $i$ regardless of the type of the mathematical formulation followed, and $n$ is the total number of design variables the problem. $h_{j}\left(x_{i}\right)$ represents nonlinear equality constraints, and ne is the total number of such constraints. $g_{j}\left(x_{i}\right)$ represents a nonlinear inequality constraint $j$. nc is the total number of constraints in the design problem. The objective function and constraints functions need not be convex. It is assumed that the region defined by (5a), (5b), and (5c) is bounded. This nonlinear programming problem is locally approximated into a linear programming problem by taking two terms of Taylor expansion of every nonlinear function in (5a), (5b), and (5c) about the current design point $x^{k}$. To assure that the approximation is adequate, the region of permissible solutions must be kept small. This is accomplished by applying move limits to the design variables.

Assuming that all the constraints are nonlinear, the programming problem (5a), (5b), and (5c) can be linearized about $x^{k}$ which is the vector of design variables $x^{k}=$ $\left\{\begin{array}{ll}x_{1}^{k} & x_{2}^{k} \cdots x_{n}^{k}\end{array}\right\}$ as

$$
\begin{array}{cc}
\text { Min } & W=w\left(x^{k}\right)+\nabla w\left(x^{k}\right)\left(x^{k+1}-x^{k}\right), \\
\text { subject to } \quad & h_{j}\left(x^{k}\right)+\nabla h_{j}\left(x^{k}\right)\left(x^{k+1}-x^{k}\right)=0, \\
j=1, \ldots, \mathrm{ne}, \\
\\
g_{j}\left(x^{k}\right)+\nabla g_{j}\left(x^{k}\right)\left(x^{k+1}-x^{k}\right) \leq 0, \\
j=n+1, \ldots, \mathrm{nc}, \\
(1-m) x^{k} \leq x^{k-1} \leq(1+m) x^{k},
\end{array}
$$

where the value of every function is known at the design point $x^{k}$, and $\nabla w\left(x^{k}\right), \nabla h_{j}\left(x^{k}\right)$, and $\nabla g_{j}\left(x^{k}\right)$ are $(1 \times n)$ gradient vectors of the functions at $x^{k}$. The last constraint in (6) defines the region of permissible solutions that is constructed by using some preselected percentage of the current design point. $m$ is known as move limit which is $0 \leq m \leq 1$. The gradient vector $\nabla w\left(x^{k}\right)$ consists of the first derivatives of function $w\left(x^{k}\right)$ :

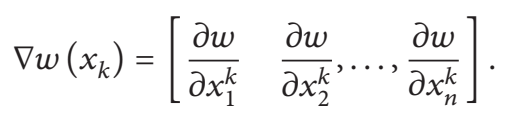

The linear programming problem (6) can be arranged in the following form:

$$
\begin{array}{ll}
\text { Min. } & W=C x^{k+1}-C_{0}, \\
\text { subject to } & A x^{k+1}\left(\begin{array}{l}
= \\
\leq
\end{array}\right) B,
\end{array}
$$

where $C_{o}$ is a constant, $C$ is a vector of order $(1 \times n)$, coefficient matrix $A$ is $[(\mathrm{nc}+2 n) \times n]$, and the right-hand side vector is of order of $[(n c+2 n) \times 1]$. They have the following form:

$$
\begin{gathered}
C_{o}=w\left(x^{k}\right)-\nabla w\left(x^{k}\right) x^{k}, \\
\mathbf{C}=\nabla w\left(x^{k}\right), \\
{[A]=\left[\begin{array}{c}
\nabla h_{j}\left(x^{k}\right) \\
\nabla g_{j}\left(x^{k}\right) \\
\mathrm{I} \\
\mathrm{I}
\end{array}\right], \quad[B]=\left[\begin{array}{c}
\nabla h_{j}\left(x^{k}\right) x^{k}-h_{j}\left(x^{k}\right) \\
\nabla g_{j}\left(x^{k}\right) x^{k}-g_{j}\left(x^{k}\right) \\
(1+m) x^{k} \\
(1+m) x^{k}
\end{array}\right],}
\end{gathered}
$$

which are constructed at current design point $x^{k}$.

The problem (8) is the linear approximation of the original nonlinear programming problem of (5a), (5b), and (5c). The simplex method can now be employed for the solution of this problem to obtain $x^{k+1}$. The previous design vector $x^{k}$ is replaced by $x^{k+1}$, and the linearization is carried out about this new point. This process is repeated with gradually decreasing move limits until no significant improvement occurs in the value of the objective function. It was found reasonable to start with $m=1$ and reduce to 0.1 each cycle until it reaches $0.1[3,5,20]$. Reinschmidt et al. [21], Pope [22] and Johnson, and Brotton [23] are some of the studies which were based on this linearization technique. In all these works, the cross-sectional properties of members were the only design variables. Saka $[3,5]$ used the linearization technique and treated joint displacements as design variables in addition to cross-sectional areas. Such formulation has also been used by Arora and Haug [24].

Sequential linear-programming-based design algorithms are attractive due to the availability of reliable linear programming packages to most of the computer users. Initial design point required to be selected by user prior to employing the algorithm can be feasible or infeasible. However, the method has a number of disadvantages. Firstly, it requires several optimization cycles to reach the optimum solution which makes it computationally costly. Secondly, choice of move limits is important because it affects the total number of iterations to find the optimum solution. Unfortunately the selection of these limits is problem dependent. Thirdly, when the starting point is infeasible, linearized problem may not have a feasible solution which means that intermediate solutions are practically useless. Furthermore, the convergence difficulties arise in the design of steel frames with large numbers of design variables. In such problems, linearization errors become large, and the linearized problem may not have a feasible solution. In both cases, it becomes necessary to relax the move limits and restart of the application of the method $[7,10]$.

Sequential quadratic programming also uses the concept of linearization, and it is one of the most effective mathematical programming techniques for nonlinearly constrained optimization problems $[12,25,26]$. The method consists of approximating the original nonlinearly constrained problem 
with a quadratic subproblem and solving the subproblem successively until convergence has been achieved on the original problem [27]. It is shown in [28] that sequential quadratic programming is quite efficient in obtaining the solution of large-scale structural optimization problems.

Wang and Arora [29] have presented two alternative formulations based on the concept of simultaneous analysis and design (SAND) for optimum design of framed structures. Nodal displacements and member forces are treated as design variables in addition to member cross-sectional properties. This leads to having stiffness equations as equality constraints in the mathematical model. The objective function and other constraints are expressed as explicit functions of the optimization variables. A sequential quadratic programming method is used to obtain the solution of the design problem. It is concluded that the SAND formulation works quite well for optimization of framed structures.

Wang and Arora [30] studied the sparsity features of simultaneous analysis and design (SAND) type of mathematical modeling for large-scale structures. It is stated that although SAND formulation has a large number of design variables gradients of the functions, and Hessian of the Lagrangian are quite sparsely populated. This property of the formulation is exploited with optimization algorithms with sparse matrix capability such as sequential quadratic programming (SQP) for solving large-scale problems. It is concluded that in terms of the wall-clock time or the CPU time/iteration, the SAND formulations are more efficient than the conventional formulation due to the fact that the number of call-to-analysis routine was much smaller.

3.1.2. Penalty Function Methods. The second group of algorithms uses sequential unconstrained minimization techniques of nonlinear programming methods to obtain the solution of the design problem. These methods replace the constrained nonlinear programming problem by a new uncontained one so that one of the unconstrained minimization algorithms can be utilized for its solution. Unconstrained minimization methods are quite general and efficient compared to constrained minimization algorithms. Thus, the basic idea is to place a penalty on constraint violation so that the solution is forced to satisfy the constraint functions. Most of the work in this area is based on the development introduced by Carroll [31] and Fiacco and McCormack [32]. These methods are widely used in structural design and have some practical advantages. There are two types of penalty function methods: exterior and interior penalty functions.

Exterior penalty function method transforms the constrained programming problem (5a), (5b), and (5c) into an uncontained one by the following function:

$$
\begin{aligned}
\operatorname{Min} \phi(x, r)= & W(x)+r \sum_{j=1}^{\mathrm{ne}} h_{j}^{2}(x) \\
& +\frac{1}{r} \sum_{j=\mathrm{ne}+1}^{\mathrm{nc}}\left\{\min \left[0, g_{j}(x)\right]\right\}^{2},
\end{aligned}
$$

for $r=1,0.1,0.01,0.001, r_{i} \rightarrow 0, r_{i}>0$, and so forth. Each penalty is directly associated with the corresponding constraint violation. The inequality terms are treated differently from the equality terms because the penalty applies only for constrained violation. The positive multiplier $r$ controls the magnitude of the penalty terms. The problem is solved by decreasing $r$ in successive steps. In exterior penalty function methods all intermediate solutions lie in the feasible region. The solution may be started from an infeasible point, which provides flexibility for the designer eliminating the need for finding the initial feasible design point. However, the algorithm cannot find a feasible solution before reaching the optimum. All intermediate solutions are infeasible and do not provide a usable design.

Interior penalty function method is employed when only inequality constraint is present in the problem. It has the following form:

$$
\phi(x, r)=W(x)+r \sum_{j=1}^{\mathrm{nc}} \frac{1}{g_{j}(x)}
$$

or in a logarithmic form

$$
\begin{gathered}
\phi(x, r)=W(x)-r \sum_{j=1}^{\mathrm{nc}} \log _{e}\left[g_{j}(x)\right], \\
r=1,0.1,0.01, \ldots \longrightarrow 0, r_{i}>0
\end{gathered}
$$

The interior penalty function method requires feasible initial design point to start with. This provides an advantage over exterior penalty method such that all intermediate solutions are feasible hence provide usable designs. Furthermore, the constraints become critical only near the end of the solution process. This provides advantage to the designers in selecting the near optimal solution, which is a less critical design instead of optimal design.

The structural optimization algorithms based on the penalty function methods are numerically reliable for problems of moderate complexity. They are general and suitable for various optimum structural design formulations. However, they have the drawback of requiring large number of structural analysis which is computationally expensive. Numerical difficulties may also arise in the case of illconditioned minimization problems. In the field of optimum structural design these methods have been used by Kavlie and Moe [33, 34], Griswold and Moe [35], and De Silva and Grant [36].

3.1.3. Gradient Method. The third group of techniques approach to the solution of a nonlinear programming problem in a direct manner. Instead of transforming the problem into another form, they deal with the constrained one as it is. These methods approach to the optimum solution step by step. At each step solution moves from current values of design variables to the next values along a suitable direction. This direction is determined by making use of the gradient vectors of the objective and constraint functions. This is why they are called gradient methods. The gradient-projection method proposed by Rosen [37] for linear constraints is based on a projecting search into the subspace defined by the active constraints. This method has been modified by 
Haug and Arora [38] for nonlinear constraints and used to develop a structural optimization algorithm. The method of feasible direction proposed by Zoutindijk [39] is employed by Vanderplaats $[40,41]$ in the popular CONMIN program. The feasible direction method starts with some design point $x^{\circ}$ at the boundary of the feasible region and then searches for the best direction and step size to move within the feasible region, to a new point $x^{1}$ such that the objective function values are improved:

$$
x^{1}=x^{\circ}+\alpha S^{\circ}
$$

where $\alpha$ is a step size and $S^{\circ}$ is the direction vector. In determining the direction vector, two conditions must be satisfied. The first is that the direction must be feasible. This is achieved in problems where the constraints at a point curve inward, if $S^{T} \nabla g_{\mathrm{j}}<$ o. If a constraint is linear or outward curved, it may require $S^{T} \Delta g_{j} \geq 0$. The second condition is that the direction must be usable, and the value of objective function is improved. This is satisfied if $S^{T} \nabla f<0$.

Despite not being very widely used in structural optimization, there are other nonlinear programming techniques that are employed in solving the steel frame design problem. Among these geometric programming [42] is applied to obtain optimum design of a number of different structural problems [43]. On the other hand, dynamic programming [44] was used in the optimum design of continuous beams and multistory frames by Palmer $[45,46]$. Both methods are applicable to problems with specialized form. This is why these techniques have not been used extensively in the field of steel frame design.

It can be concluded that mathematical programming techniques are general. It is possible to include displacement stress, stiffness, and instability constraints in the optimum design formulation of the framed structures. Inclusion of stability constraints results in highly nonlinear programming problem. Among the large number of available methods $[47,48]$ the techniques used in the structural optimization can be collected in three groups. The literature survey shows that the optimum design algorithms developed are good only in designing structures with few members. Unfortunately, there is no single general nonlinear programming algorithm that can effectively be used in solving all types of structural optimization problems. Those that perform well in small- or moderate-size structures run into numerical difficulties in large-size structures. Except few, computer implementation of these algorithms mostly is cumbersome. They require several structural analyses to be carried out in each design cycle to update the response of the structure. This makes them computationally expensive. Overall, it is reasonable to state that among the existing mathematical programming methods, the ones that make use of approximation techniques such as sequential quadratic programming result in an efficient structural optimization algorithms even for largescale optimum design problems if the design variables are continuously not discrete.

3.2. Optimality Criteria Methods Based on Steel Frame Design Optimization Techniques. Optimality criteria methods differ from the mathematical programming methods in the way they solve the optimum design problem. While mathematical programming techniques attempt to minimize the objective function directly considering the constraint functions, the optimality criteria methods derive a criterion based on intuition such as fully stressed design or based on a mathematical statement such as Kuhn-Tucker conditions. They then establish an iterative procedure to achieve this criterion. It is expected that when this is accomplished, the optimum solution will be obtained. Optimality criteria methods are not as general as the mathematical programming techniques. However, they are computationally more efficient. Their performance does not depend on the size of the design problem, and they are easier to implement for computers. Number of structural analysis required to reach the final design is much less than mathematical programming methods. However, in certain cases, they may not converge to the optimum solution.

Optimality criteria methods were originated by Prager and his coworkers [49] for continuous systems using uniform energy distribution criteria. Venkayya et al. [50-52], Berke [53, 54], and Khot et al. [55-57] have later applied this idea to discrete systems. Ragsdell has reviewed these techniques in [58]. In these methods, a prior criterion is derived using Kuhn-Tucker conditions. This criterion that is required to be satisfied in the optimum solution provides a basis in producing a recursive relationship for design variables. This relationship is used to resize the structural elements during the optimum design cycles. The design process is terminated when convergence is attained in the value of objective function. The optimality criteria method is applied to optimum design of pin-jointed structures by Feury and Geradin [59], Fleury [60], and Saka [61]. It is applied to optimum design of steel framed structures by Tabak and Wright [62], Cheng and Truman [63], Khan [64], and Sadek [65]. Khot et al. [55] have carried out the comparison of different optimality criteria methods. In all these algorithms, displacement, stress, and minimum-size constraints on the design variables were all considered. It is shown in these works that the design methods based on the optimality criteria approach are straightforward and easy to program. Their behavior in obtaining the optimum solution does not change depending on the number of design variables. Number of structural analysis required to reach to optimum design is relatively small compared to mathematical programming based techniques which makes optimality criteria based structural optimization algorithms efficient and attractive for practicing engineers.

However, none of the previously mentioned methods have considered the code specifications in the formulation of the design problem. Saka [66] has presented optimality criteria based minimum weight design algorithm for pin jointed steel structures where the design requirements were the same as the ones specified in AISC [13]. In this work, an algorithm is developed for the optimum design of pin-jointed structures under the number of multiple loading cases while considering displacements, stress, buckling, and minimumsize constraints. In contrast to the previous work, fixedvalue allowable compressive stresses were not utilized for the compression members; instead they are left to the algorithm 
to be decided. The values of critical stresses are computed in every design step by making use of the current values of the design variables. In order to achieve this, the critical stresses are first expressed in terms of design variables as nonlinear linear equations by making use of the relationships given in design codes. These equations are then solved by the NewtonRaphson method to obtain the value of the design variable that satisfies the buckling constraint.

3.2.1. Linear Elastic Steel Frames. The optimum design problem of a rigidly jointed plane frame formulated according to ASD-AISC (Allowable Stress Design, American Institute of Steel Construction) [13] can be expressed as follows:

$$
\begin{aligned}
& \text { Min } \quad W=\sum_{k=1}^{\mathrm{ng}} A_{k} \sum_{i=1}^{m k} \rho_{i} \ell i, \\
& \text { Subject to } \quad \delta_{j \ell} \leq \delta_{j \ell u}, \quad j=1, \ldots, p \text {, } \\
& \ell=1, \ldots, \mathrm{n} \ell \mathrm{c}, \\
& \frac{f_{\mathrm{anl}}}{F_{\mathrm{anl}}}+\frac{m_{1}}{m_{2}} \frac{f_{\mathrm{bxnl}}}{F_{\mathrm{bxnl}}} \leq 1, \quad n=1, \ldots, \mathrm{nm}, \\
& A_{k} \geq A_{k \ell}, \quad k=1, \ldots, \mathrm{ng},
\end{aligned}
$$

where $A_{k}$ is the area of members belonging to group $k$; $m k$ is the total number of members in group $k ; \rho_{i}$ and $\ell_{i}$ are the density of the material and the length of members in group $i$; ng is the total number of member groups in the structure. $\delta_{j \ell}$ is the displacement of joint $j$ under the load case $\ell$, and $\delta_{j \ell u}$ is its upper bound; $p$ is the total number of restricted displacements. $\mathrm{n} \ell \mathrm{c}$ is the total number of load cases that the frame is subjected to. $f_{\text {anl }}$ and $f_{\text {bxnl }}$ are the computed axial stress and compressive bending stress in member $n$ under load case $\ell . F_{\text {anl }}$ is the allowable axial compressive stress considering that the member is loaded by axial compression only, $F_{\text {bxnl }}$ is the allowable compressive bending stress considering that the member $i$ is loaded in bending only. $m_{1}$ is a constant and $m_{2}$ is an expression given in [13]. $\mathrm{nm}$ is the total number of members in the frame. $A_{k \ell}$ is the lower bound on the design variable $A_{k}$. This bound is required to prevent member areas being reduced to zero in the optimum solution.

In the optimum design problem (14) only the crosssectional areas of different groups in the frame are treated as design variables. It is apparent that determination of the response of the frame under the applied loads necessitates the values of the other cross-sectional properties such as moment of inertia and sectional modulus. Therefore it becomes necessary to use the relationship (3) to relate the other crosssectional properties to sectional areas in order not to increase the number of design variables in the programming problem.

The solution of the design problem (14) is obtained in an iterative manner. The area variables are changed during iterations with the aim of obtaining a better design. It is apparent that the new values of design variables are decided by the most severe design constraint in every design cycle. This necessitates obtaining an expression for updating these variables depending upon whether the displacement, stress, or the buckling constraints are dominant in the design problem. If neither of these constraints is dominant, then the area variable is decided by the minimum-size limitation.

Dominance of Displacement Constraints. In the case where the displacement constraints are dominant in the design problem, the new values of the design variables are decided by these constraints. If the design problem (14) is rewritten by only considering the displacement constraints, the following mathematical model is obtained:

$$
\begin{array}{ll}
\operatorname{Min} & W=\sum_{K=1}^{\mathrm{ng}} A_{k} \sum_{i=1}^{m k} \rho_{i} \ell_{i}, \\
\text { subject to } \quad g_{i \ell}\left(A_{k}\right)=\delta_{j \ell}-\delta_{j \ell u} \leq 0, \quad j=1, \ldots, p, \\
& \ell=1, \ldots, \mathrm{n} \ell \mathrm{c} .
\end{array}
$$

Employing language multipliers, this constrained problem can be transformed into unconstrained form as

$$
\phi\left(A_{k}, \lambda_{j \ell}\right)=\sum_{k=1}^{\mathrm{ng}} A_{k} \sum_{j=1}^{m k} \rho_{i} \ell_{i}+\sum_{\ell=1}^{\mathrm{n} \ell} \sum_{J=1}^{P} \lambda_{j \ell} g_{j \ell}\left(A_{k}\right),
$$

where $\lambda_{j \ell}$ is the Lagrangian parameter for the $j$ th constraint under the $\ell$ th load case. The necessary conditions for the local constrained optimum are obtained by differentiating (16) with respect to the design variable $A_{k}$ as

$$
\frac{\partial \phi\left(A_{k}, \lambda_{j \ell}\right)}{\partial A_{k}}=\sum_{j=1}^{m k} \rho_{i} \ell_{i}+\sum_{\ell=1}^{\text {nce }} \sum_{J=1}^{P} \lambda_{j \ell} \frac{\partial g_{j \ell}\left(A_{k}\right)}{\partial A_{k}}=0 .
$$

By using the virtual work method $\delta_{j l}$, the $j$ th displacement of the frame under the load case $\ell$ can be expressed as

$$
\delta_{j \ell}=X_{\ell}^{T} K X_{j},
$$

where $X_{j}$ is the virtual displacement vector due to virtual loading corresponding to the $j$ th constraint. This loading case is setup by applying a unit load in the direction of the restricted displacement $j . K$ is the overall stiffness matrix of the frame, and $X_{\ell}$ is the displacement vector due to the applied load case $\ell$. Equation (18) can be decomposed as sum of the contributions of each member in the frame as

$$
\delta_{j l}=\sum_{i=1}^{\mathrm{nm}} X_{i l}^{T} K_{i} X_{i j}=\sum_{i=1}^{\mathrm{nm}} \frac{f_{i j l}}{A_{i}},
$$

where $f_{i j l}$ is known as the flexibility coefficient

$$
f_{i j l}=X_{i l}^{T} K_{i} X_{i j} A_{i}
$$

where $K_{i}$ is the contribution of member $i$ to the overall stiffness matrix. Substituting (20) into the derivative of the displacement constraint leads to the following:

$$
\frac{\partial g_{j \ell}\left(A_{k}\right)}{\partial A_{k}}=-\frac{1}{A_{k}^{2}} \sum_{j=1}^{m k} f_{i j \ell},
$$


which only involves with the members in group $k$. Hence (17) becomes

$$
\sum_{i=1}^{m k} \rho_{i} \ell_{i}-\sum_{\ell=1}^{n \ell c} \sum_{j=1}^{p} \lambda_{j \ell} \frac{1}{A_{k}^{2}} \sum_{j=1}^{m k} f_{i j \ell}=0 .
$$

Rearranging (22), considering constant $\rho$ throughout the frame, and multiplying both sides by $A_{k}^{r}$ and then taking the $r$ th root leads to

$$
\begin{array}{r}
A_{k}^{v+1}=A_{k}^{v}\left[\frac{1}{\rho \sum_{i=1}^{m k} l_{i}} \sum_{\ell=1}^{n \ell c} \sum_{j=1}^{P} \frac{\lambda_{j l}}{A_{k}^{2}} \sum_{j=1}^{m k} f_{i j \ell}\right]^{1 / r}, \\
k=1, \ldots, \mathrm{ng},
\end{array}
$$

where $v$ is the iteration number. This is known as the optimality criterion. It can be used to obtain the new values of design variables in each iteration provided that Lagrange parameters are known. There are several suggestions for the computation of Lagrange parameters. They are summarized in [55]. Among them, the one suggested by Khot [56] is simple and easy to apply:

$$
\lambda_{j \ell}^{v+1}=\lambda_{j \ell}^{v}\left(\frac{\delta_{j \ell}}{\delta_{j \ell u}}\right)^{1 / c}, \quad j=1, \ldots, p,
$$

where $v$ is the current and $v+1$ is the next iteration number. $c$ is a preselected constant known as a step size. The value of 0.5 provides steady convergence. It is apparent that in order to use (24), it is necessary to select some initial values for Lagrange multipliers.

Dominance of Strength Constraints. In the case where the strength constraints are dominant in the design problem it becomes necessary to derive an expression from which the new values of area variables can be computed. In this study this expression is based on ASD-AISC [13] inequalities which are repeated below for only considering in-plane bending:

$$
\begin{aligned}
\frac{f_{a}}{F_{a}}+\frac{C_{m x} f_{b x}}{\left(1-f_{a} / F_{e x}^{\prime}\right) F_{b x}} & \leq 1, \\
\frac{f_{a}}{0.6 F_{y}}+\frac{f_{b x}}{F_{b x}} & \leq 1,
\end{aligned}
$$

where $x$ with subscripts $b, m$, and $e$ is the axis of bending which a particular stress or design property applies, $F_{y}$ is the yield stress, and $f_{a}$ and $f_{b}$ are the computed axial stress and bending stress at the point under consideration. $F_{a}$ is the allowable axial compressive stress considering that the member is only axially loaded, $F_{b}$ is the allowable compressive bending stress considering that the member is only under bending loading. $C_{m}$ is a factor and is given as 0.85 for compression members in frames subject to joint translation. $F_{e x}^{\prime}$ is Euler stress divided by a factor:

$$
F_{e x}^{\prime}=\frac{12 \pi_{2} E}{23 S^{2}},
$$

where $S=\eta l_{b} / r_{b}$ is the slenderness ratio of member, $l_{b}$ is the actual unbraced length in the plane of bending, $r_{b}$ is the corresponding radius of gyration, $\eta$ is the effective length factor in plane of bending. and $E$ is the modulus of elasticity.

The inequalities (25) and (26) are required to be satisfied for those frame members subjected to both axial compression and bending; the others that are subjected to axial tension and bending require satisfying the inequality (26). In these inequalities the allowable stresses $F_{a}, F_{b}$, and $F_{e}$ are related to the instability of the member. They are function of various cross-sectional properties that are to be expressed in terms of area variables.

Axial Stability Constraints. The allowable critical stress $F_{a}$ for a compression member $i$ under load case $\ell$ is given in ASDAISC as the following.

If $S_{i} \geq C$ elastic buckling is

$$
F_{a}=\frac{12 \pi^{2} E}{23 S_{i}^{2}} .
$$

If $S_{i} \leq C$ plastic buckling is

$$
\begin{aligned}
F_{a} & =\frac{F_{y}\left(1-S_{i}^{2} / 2 C^{2}\right)}{n}, \\
n & =\frac{5}{3}+\frac{3}{8} \frac{S_{i}}{C}-\frac{S_{i}^{3}}{8 C^{3}},
\end{aligned}
$$

where $S_{i}$ is the slenderness ratio of the member $i$ and $C$ is given as $\sqrt{\left(2 \pi^{2} E / F_{y}\right)}$. It is apparent from (28) and (29) that critical stress of a compression member is function of its slenderness ratio which in turn is related to the radius of gyration of the cross section of a member. The crosssectional areas change from one design step to another as well as from one member to another during the design process. This results in having varying critical stress limits in the design optimization problem of the steel frame. Consequently it becomes necessary to express the allowable critical stress of a compression member in terms of area variables so that its value can be calculated whenever the cross-sectional area of a member changes. This is achieved by employing the approximate relationship given in (3). Computation of allowable critical stress of a compression member differs depending on its slenderness ratio as given in (28) and (29). It is found more suitable here to identify whether a compression member buckles in elastic region or in plastic region through the value of the critical load $P_{\mathrm{cr}}$. This load is obtained by using the fact that for the value of $S_{i}=C(28)$ and (29) give the same value. That is

$$
S_{i}=C ; \quad P_{\mathrm{cr}}=F_{a} A_{\mathrm{cr}} ; \quad P_{\mathrm{cr}}=\frac{12 \pi^{2} E A_{\mathrm{cr}}}{23 C},
$$

where $A_{\mathrm{cr}}$ is the critical area value for member $i$. Since a compression member can buckle about its $x-x$ or $y-y$ axis depending on whichever slenderness ratio is critical, it is then necessary to express both radii of gyration in terms of area variables by using the relationship

$$
r_{x}=a_{1}^{0.5} A^{c_{1}}, \quad r_{y}=a_{2}^{0.5} A^{c_{2}},
$$


where $c_{1}=0.5\left(b_{1}-1\right)$ and $c_{2}=0.5\left(b_{2}-1\right)$. It follows from $s_{x i}=l_{x i} / r_{x i}$ and $S_{y i}=l_{y i} / r_{y i}$ where $l_{x i}$ and $l_{y i}$ are the effective length of member $i$ about $x-x$ and $y-y$ axis, respectively. The critical area value is then easily obtained from

$$
S_{i}=\frac{l_{k}}{\left(a_{k}^{0.5} A_{\mathrm{cr}}^{c_{k}}\right)} ; \quad A_{\mathrm{cr}}=\left[\frac{l_{k}}{\left(a_{k}^{0.5} C\right)}\right]^{c_{k}},
$$

where the subscript $k$ of $l_{k}$ is either $x$ or $y$ and subscript $k$ of $a_{k}$ and $c_{k}$ is either 1 or 2 , whichever applies.

After the computation of $P_{\text {cr }}$ from (37), the check for the elastic or plastic buckling can proceed as the following.

If $P_{a} \leq P_{\mathrm{cr}}$ elastic buckling is

$$
F_{a}=\frac{12 \pi^{2} E}{23 S_{i}^{2}}
$$

If $P_{a} \geq P_{\mathrm{cr}}$ plastic buckling is

$$
F_{a}=\frac{F_{y}\left(24 C^{3}-12 C S_{i}^{2}\right)}{40 C^{3}+9 S_{i} C^{2}-3 S_{i}^{3}}
$$

where $P_{a}$ is the compression force in member $i$ under the load case $\ell$.

Finally the previously mentioned allowable stresses can be related to area variables by substituting $S_{i}=l_{k} /\left(a_{k}^{0.5} A^{c_{k}}\right)$ which yields to the following expressions.

If $P_{a} \leq P_{\text {cr }}$ elastic buckling is

$$
F_{a}=t_{6} A^{2 c_{k}}
$$

If $P_{a} \geq P_{\text {cr }}$ plastic buckling is

$$
F_{a}=\frac{e_{1} A^{3 c_{k}}-e_{2} A^{c_{k}}}{e_{3} A^{3 c_{k}}+e_{4} A^{2 c_{k}}-e_{5}}
$$

where the constants are

$$
\begin{gathered}
t_{6}=\frac{12 \pi^{2} E a_{k}}{\left(23 l_{k}^{2}\right)}, \quad e_{1}=24 F_{y} C^{3} a_{k}^{1.5}, \quad e_{2}=12 F_{y} l_{k}^{2} C a_{k}^{0.5}, \\
e_{3}=40 C^{3} a_{k}^{1.5}, \quad e_{4}=9 C^{2} l_{k} a_{k}, \quad e_{5}=3 l_{k}^{3} .
\end{gathered}
$$

In the previously mentioned expressions

$$
\begin{aligned}
& \text { if } S_{x i} \leq S_{y i} \text { then } l_{k}=l_{y i}, a_{k}=a_{2}, c_{k}=c_{2}, \\
& \text { if } S_{x i}>S_{y i} \text { then } l_{k}=l_{x i}, a_{k}=a_{1}, c_{k}=c_{1} .
\end{aligned}
$$

Consequently, the allowable axial compressive stress $F_{a}$ of inequality (25) is replaced by either (35) or (36), whichever applies.

Lateral Stability Constraints. The allowable compressive stress $F_{b}$ of (25) and (26) is given by the following formulae in ASDAISC:

$$
\begin{aligned}
& \text { when } \sqrt{\frac{70830 C_{b}}{F_{y}}} \leq \frac{l_{e}}{r_{t}} \leq \sqrt{\frac{354200 C_{b}}{F_{y}}} \\
& \text { then } F_{b}=\left[\frac{2}{3}-\frac{F_{y}\left(l_{e} / r_{t}\right)^{2}}{1.055 \times 10^{6} C_{b}}\right] F_{y},
\end{aligned}
$$

$$
\text { when } \frac{l_{e}}{r_{t}}>\sqrt{\frac{354200 C_{b}}{F_{y}}} \text { then } F_{b}=\frac{1.17 \times 10^{5} C_{b}}{\left(l_{e} / r_{t}\right)^{2}} \text {, }
$$

where the units of the yield stress $F_{y}$ and the allowable compressive bending stress $F_{b}$ are $\mathrm{kN} / \mathrm{cm}^{2}$.

In (40) and (41) the value of $F_{b}$ cannot be more than 0.6. $F_{y}$ and $r_{t}$ are the radii of gyration of I section comprising the flange plus one-third of the compression web area taken about minor axis. $l_{e}$ is the lateral effective length of the beam. The coefficient $C_{b}$ is given a

$$
C_{b}=1.75+1.5 \beta+0.3 \beta^{2} \leq 2.3
$$

in which $\beta$ is the ratio of the small moment $M_{1}$ to the larger moment $M_{2}$ acting at the ends of the member. $\beta$ has positive sign if the member is in single curvature, has negative sign otherwise. Since the end moments of the frame members are available at every design step from the analysis of frame which is carried out at every design step, $C_{b}$ becomes a constant which makes $F_{b}$ only a function of $r_{t}$. In I shaped sections $r_{t}$ may be approximated as $1.2 r_{y}$ as given in [67]. $r_{t}$ can be expresses in terms of area variable by employing the relationship (3) as

$$
r_{t}=1.2 r_{y}=1.2 a_{2}^{0.5} A^{c_{2}}
$$

Substitution of (43) into (44) yields

$$
F_{b}=\left(t_{1}-t_{2} A^{-2 c_{2}}\right)
$$

where

$$
t_{1}=\frac{2 F_{y}}{3}, \quad t_{2}=\frac{F_{y}^{2} l_{e}^{2}}{\left(1.519 \times 10^{6} a_{2} C_{b}\right)} .
$$

And substituting in (41) gives

$$
F_{b}=t_{4} A^{2 c_{2}},
$$

where

$$
t_{4}=1.6848 \times 10^{5} a_{2} C_{b} l_{e}^{-2} .
$$


Depending on the lateral slenderness ratio of the beam either (44) or (46) is substituted in (25) and (26).

Combined Stress Constraints. The Euler stress given in (27) can also be expressed in terms of area variables by substituting $S_{i}=l_{x} /\left(a_{1}^{0.5} A^{c_{1}}\right)$ which yields to the following expression:

$$
F_{e x}^{\prime}=t_{5} A^{2 c_{1}}
$$

where

$$
t_{5}=\frac{12 \pi^{2} E a_{1}}{\left(23 l_{x}^{2}\right)} .
$$

The axial and bending stresses in the member due to the applied loads are

$$
f_{a}=\frac{P_{a}}{A}, \quad f_{b}=\frac{M_{x}}{\left(a_{3} A^{b_{3}}\right)},
$$

where $P_{a}$ and $M_{x}$ are the axial force and the maximum bending moment in the member.

Substituting (48) and (50) into (25) with $C_{m}=0.85$ and carrying out simplifications the combined stress constraint can be reduced to the following nonlinear equation:

$$
\begin{aligned}
& \alpha_{1} F_{b} A^{b_{3}}-\alpha_{2} F_{b} A^{c_{3}}+\alpha_{3} F_{a} A-\alpha_{4} F_{b} F_{a} A^{b_{3}+1} \\
& +\alpha_{5} F_{b} F_{a} A^{c_{3}+1}=0
\end{aligned}
$$

where $c_{3}=b_{3}-2 c_{1}-1$ and the constants are

$$
\begin{gathered}
\alpha_{1}=a_{3} t_{5} P_{a}, \quad \alpha_{2}=a_{3} P_{a}^{2}, \quad \alpha_{3}=0.85 M_{x} t_{5}, \\
\alpha_{4}=a_{3} t_{5}, \quad \alpha_{5}=a_{3} P_{a} .
\end{gathered}
$$

It is apparent from (35), (36), (44), and (46) that $F_{a}$ and $F_{b}$ are also nonlinear expressions of area variables. Substitution of these into (51) increases the nonlinearity of the equation even further. Similarly substitution of (50) into the combined stress constraint of (26) reduces this equation into a nonlinear equation of area variable:

$$
u_{1} F_{b} A^{b_{3}}+u_{2} A-u_{3} F_{b} A^{b_{3}+1}=0
$$

where the constants are

$$
u_{1}=a_{3} P_{a}, \quad u_{2}=0.6 F_{y} M_{x}, \quad u_{3}=0.6 F_{y} a_{3} .
$$

The equations (51) and (53) are solved using NewtonRaphson method to obtain the value of area variables that satisfy the combined stress constraints. The solution of these equations does not introduce any difficulty although they are highly nonlinear. The derivatives with respect to area variables that are required by Newton-Raphson method are evaluated analytically since $F_{a}$ and $F_{b}$ are already expressed in terms of area variables. Numerical experiments have shown that it takes not more than 5 to 6 iterations to obtain the roots of these nonlinear equations. The larger value obtained from these equations is adopted as the member area for the next design step for the case where the combined stress constraints are dominant in the design problem.

Optimum Design Algorithm. The steps of the design procedure described previously are given in the following.

(1) Select the initial values of area variables and Lagrange multipliers.

(2) Analyze the frame with these values of area variables and obtain the joint displacements as well as member end forces under the external loads and unit loads applied in the direction of the restricted displacements.

(3) Compute the Lagrange multipliers from (24).

(4) Take each area group in the frame respectively and compute the new value of the area variable which is in the cycle from (23).

(5) Compute the lower bound on the same area variable for the combined stress constraints from (51) and (53). Select the largest.

(6) Compare the three area values obtained from dominant displacement constraints, the combined stress constraints, and the minimum size restrictions. Take the largest among three as the new value of the area variable for the next design cycle.

(7) Carry out the steps 4, 5, and 6 for all the area groups in the frame.

(8) Check the convergence on the weight of the frame. If it is satisfied go to step 9; else go to step 2.

(9) Check whether the displacement and combined stress constraints are satisfied. If not then go to step 2; else stop.

The initial design point required to initiate the design procedure can be selected in any way desired. It can be feasible or even be infeasible. The area variables in the initial design point can be selected as all are equal to each other for simplicity or can be decided using engineering judgment. The numerical experimentation carried out has shown that the design algorithm works equally well with all these different initial points.

SODA developed by Grieson and Cameron [68] was the first commercial structural optimization software for practical buildings design. This software considered the design requirements from Canadian Code of Standard Practice for Structural Steel Design (CAN/CSA-S16-01 Limit States Design of Steel Structures) and obtained optimum steel sections for the members of a steel frame from an available set of steel sections. However, the software was limited to relatively small skeleton framework.

Optimality criteria based structural optimization method is presented in [69] for the optimum designs of multistory reinforced concrete structures with shear walls. The algorithm considers displacement, ultimate axial load, and bending moment and minimum size constraints. Member depths are treated as design variables. The values of design 
variables are evaluated from recursive relationships in every design cycle depending on the dominance of design constraints. The largest value of the design variable among those computed from the displacement limitations, ultimate, axial, and bending moment constraints, and minimum size restrictions defines the new value of the design variable in the next optimum design cycle. This process is repeated until convergence is obtained on the objection function.

It is shown in [70] that optimality criteria approach can also be utilized in the optimum geometry design of roof trusses. In this work the slope of upper chord of a truss is treated as a design variable in addition in to area variables. Additional optimality criteria were developed for slope variables under the displacement constraints; the algorithm determines the optimum values of the cross-sectional areas in the roof truss as well as optimum height of the apex.

In another application [71], the optimality criteria based structural optimization algorithm is developed for the optimum design of steel frames with tapered members. The algorithm considers the width of an I section as constant together with web and flange thickness, while the depth is assumed to be varying linearly between joints. The depth at each joint in the frame where the lateral restraints are assumed to be provided is treated as a design variable. The objective function taken as the weight of the frame is expressed in terms of the depth at each joint. The displacement and combined axial and flexural strength constraints are considered in the formulation of the design problem in accordance with Load and Resistance Factor Design (LRFD) of AISC code [72]. The strength constraints that take into account the lateral torsional buckling resistance of the members between the adjacent lateral restraints are expressed as a nonlinear function of the depth variables. The optimality criteria method is then used to obtain a recursive relationship for the depth variables under the displacement and strength constraints. The algorithm basically consists of two steps. In the first one, the frame is analyzed under the external and unit loadings for the current values of the design variables. In the second, this response is utilized together with the values of Lagrange multipliers to compute the new values of the depth variables. This process is continued until convergence obtained in the objective function. The optimum design of number of practical pitched roof frames is presented to demonstrate the application of the algorithm.

Al-Mosawi and Saka [73] employed optimality criteria approach to develop an algorithm for the optimum design of single-core shear walls subjected to combined loading of axial force, biaxial bending moment, and torsional moment. The algorithm is based on the limit state theory and considers displacement limitations in addition to strain constraints in concrete and yielding constraints in rebars. It takes into account the effect of warping which can be considerable in the computation of normal stresses when the thin-walled section is subjected to a torsional moment. The design algorithm makes use of sectional properties of section, which is quite useful in describing deformations and stresses when the plane cross-section no longer remains plane. A numerical procedure is developed to calculate the shear center of reinforced concrete thin-walled section in addition to the sectional and sectional properties. Furthermore, an iterative procedure is presented for finding the location of the neutral axis in reinforced concrete thin-walled sections subjected to axial force, biaxial, moments and torsional moment. It is shown that optimality criteria method can effectively be used in obtaining the optimum solution of highly nonlinear and complex design problem.

Al-Mosawi and Saka [74] presented an algorithm that obtains the optimum cross-sectional dimensions of coldformed thin-walled steel beams subjected to axial force, biaxial moments, and torsional loadings. The algorithm treats the cross-sectional dimensions such as width, depth and wall thickness as design variables and considers the displacement as well as stress limitations. The presence of torsional moments causes wrapping of thin-walled sections. The effect of warping in the calculations of normal stresses is included using Vlasov [75] theorems. The design problem turns out to be highly nonlinear problem. It is shown that the optimality criteria method can effectively be used to obtain the solution.

Chan and Grierson [76] and Chan [77] presented a practical optimization technique based on the optimality criteria approach for the design of tall steel building frameworks where cross-sectional areas are selected from the standard steel section tables. This computer based method considers the multiple interstory drift, strength, and sizing constraints in accordance with building code and fabrications requirements. The optimality criteria approach and section properties' regression relationship are used to solve the design problem. To achieve a final optimum design using standard steels section a pseudo-discrete optimality criteria technique is applied to assign standard steel sections to the members of the structure while maintaining the least change in structure weight. A full-scale 50 story three-dimensional steel frame is designed to demonstrate the application of the automatic optimal design method.

Soegiarso and Adeli [78] presented an algorithm for the minimum weight design of steel moment resisting space frame structures with or without bracing based on LRFD specifications of AISC. The algorithm is based on the optimality criteria method. The LRFD constraints for moment resisting frames are highly nonlinear and implicit functions of design variables. The structure is subjected to wind loading according to the Uniform Building Code in addition to the dead and live loads. The algorithm is applied to the optimum design of four large high-rise steel building structures ranging up in height from 20 to 81 stories.

3.2.2. Nonlinear Elastic Steel Frames. Optimality criteria approach has been effectively employed in the optimum design of nonlinear skeleton structures. Khot [79] was one of the early researchers who presented an optimization algorithm based on an optimality criteria approach to design a minimum weight space truss with different constraint requirements on system stability. In order to reduce the imperfection sensitivity of a structure, the Eigen values associated with the system buckling modes are separated by a specified interval. This requirement is included as a constraint in the design 
problem. This design obtained for the various constraint conditions was analyzed with and without specified geometric imperfections using nonlinear finite element program that accounts geometric nonlinearity. The results obtained for various designs were compared for their imperfection sensitivity. Later, Khot and Kamat [80] presented optimality criteria based algorithm for the minimum weight design of geometrically nonlinear pin-jointed space trusses. The nonlinear critical load is determined by finding the load level at which the Hessian of the potential energy becomes negative. A recurrence relationship is based on the criteria that at the optimum structure the nonlinear stain energy density must be equal in all members. This relationship is used to resize the space truss members. The number of examples is considered to demonstrate the application of the algorithm.

Saka [81] also presented an optimum design algorithm that takes into account the response of space trusses beyond the elastic behavior. The algorithm is developed by coupling a nonlinear analysis technique with an optimality criteria approach. The nonlinear analysis method used determines the changes in the axial stiffness of space truss members from the nonlinear load-deformation curves. These curves are obtained from the tensile stress-strain curve for the tension members and from the stress-strain curve under compression that varies as the slenderness ratio of the member changes. These nonlinear curves are approximated by straight lines. The intersection points of these lines are called as critical points. As the load increases, the slope of linear segments changes. This implies the variation in the axial stiffness of the member. Once the axial stiffness values of all members are specified up to the failure, the nonlinear analysis is easily carried out by allowing these changes in the stiffness of members during the increase of the external loads. The optimality criteria approach is employed to obtain a recurrence relationship for area variables. Consideration of postbuckling and postyielding behavior of truss members makes the stress and buckling constraints redundant. The number of space trusses is designed by the algorithm, and it is shown that optimum designs are obtained after relatively fewer members of iterations.

Saka and Ulker [82] developed a structural optimization algorithm for geometrically nonlinear space trusses subjected to displacement and stress and size constraints. Tangent stiffness method is used to obtain the nonlinear response of the space truss. This response is used by the optimality criteria method to determine the values of area variables in the next design cycle. During the nonlinear analysis, tension members are loaded up to yield stress, and compression members are stressed until their critical limits. The overall loss of elastic stability is checked throughout the steps of the algorithm. It is shown that the consideration of geometric nonlinearity in the optimum design of space trusses makes it possible to achieve further reduction in its overall weight. It is shown that inclusion of the geometric nonlinearity caused $11 \%$ reduction in the overall weight in the optimum design of 120-bar laminar dome compared to minimum weight design considering linear behavior. Furthermore, by considering the geometrical nonlinearity in the optimum design the algorithm takes into account the realistic behavior of space trusses. Geometric nonlinearity becomes important in shallow-framed domes.

Saka and Hayalioglu [83] present a structural optimization algorithm for geometrically nonlinear elastic-plastic frames. The algorithm is developed by coupling the optimality criteria approach with large deformation analysis method for elastic-plastic frames. The optimality criteria method is used to obtain a recursive relationship for the design variables considering the displacement constraints. The nonlinear response of the frame used by the recursive relationship is based on a Euclidian formulation which includes elasticplastic effects. Local member force-deformation relationships are extended to cover the geometric nonlinearities. An incremental load approach with Newton-Raphson iterations is adopted for the computational procedure. These iterations are terminated when the prescribed load factor reached. It is shown in the optimum design of number of rigid frames considered in the study that inclusion of geometric and material nonlinearity in the optimum design does not only lead to a more realistic approach but also yields to lighter frames. The reduction in the overall weight of the frames varied from $20 \%$ to $30 \%$ when compared to the linear-elastic optimum frame designs. Later, the algorithm is extended to geometrically nonlinear elastic-plastic steel frames with tapered members [84]. It is shown that consideration of nonlinear behavior in the minimum weight design of pitched roof frame with tapered members yields almost $40 \%$ reduction in the weight compared to the optimum design with linear-elastic behavior. It is stated in both works that the optimality criteria approach was quite effective in handling the displacement constraints in such complex design problems. It was noticed that most of the computational time was consumed by the large deformation analysis of elastic-plastic frame.

Saka and Kameshki [85] employed optimality criteria approach to develop an algorithm for the optimum design of unbraced rigid large frames that takes into account the nonlinear response of $\mathrm{P}-\delta$ effect. The algorithm considers sway constraints and combined stress limitations in the design problem. A recursive relationship is developed for using optimality criteria approach for the sway limitations and the combined strength constraints are reduced to nonlinear equations of the design variables. The algorithm initiates the design process by carrying out nonlinear analyses of the frame in which in each analysis cycle the overall stability is checked. When the nonlinear response of the frame is obtained without loss of stability, the new values of design variables are computed from the recursive relationships. This process of reanalyses and resizing is repeated until the convergence is obtained in the objection function. It was noticed that the nonlinear value of the top storey sway of 24story 3-bay unbraced frame due to P- $\delta$ effect was $10 \%$ more than its linear value. This clearly indicates the importance of including the geometric nonlinearity in the optimum design of unbraced tall steel frames.

This algorithm is later extended to the optimum design of three-dimensional rigid frames by Saka and Kameshki [86]. The algorithm considers the displacement limitations 
and restricts the combined stresses not to be more than yield stress. The stability functions for three-dimensional beamcolumns are used to obtain the $\mathrm{P}-\delta$ effect in the frame. These functions are derived by considering the effect of axial force on flexural stiffness and effect of flexure on axial stiffness. The algorithm employs the optimality criteria approach together with nonlinear overall stiffness matrix to develop a recursive relationship for design variables in the case of dominant displacement constraints. The combined stress constraints are reduced to nonlinear equations of design variables. The algorithm initiates the optimum design at the selected load factor and carries out elastic instability analysis until the ultimate load factor is reached. During these iterations checks of the overall stability of the space rigid frame are conducted. If the nonlinear response is obtained without loss of stability, the algorithm proceeds to the next design cycle. It is shown in the design examples considered that $\mathrm{P}-\delta$ effect plays important role in the optimum design of framed domes, and its consideration does not only provide more economy in the weight, but also produces more realistic results.

The research work reviewed previously clearly shows that the optimality criteria approach is quite effective in obtaining the optimum design of skeleton structures. The number of design cycles required to reach to the optimum frame is relatively low and it is independent of the size of frame. The optimality criteria approach made it possible to obtain the optimum design of large size, realistic structures. It is also shown that these methods are general and can be employed in the optimum design of linear elastic, nonlinear elastic, and elastic-plastic frames. Furthermore it is shown that they can even be used in the shape optimization of skeleton structures. Thus, it is apparent that in structural engineering problems where the design variables can have continuous values, the optimality criteria based structural optimization algorithm effectively provides solutions. However, the optimum design of steel frames necessitates selection of steel profiles for its members from available list of steel sections which contains discrete values not continuous. Altering optimality criteria algorithms to cater this necessity is cumbersome and do not yield techniques that can be efficiently used in the optimum design of large-size steel frames. This discrepancy of mathematical programming and optimality criteria based design optimization algorithms forced researchers to come up with new ideas which caused the emergence of stochastic search techniques.

\section{Discrete Optimum Design Problem of Steel Frames to LRFD-AISC}

The design of steel frames requires the selection of steel sections for its columns and beams from a standard steel section tables such that the frame satisfies the serviceability and strength requirements specified by LRFD-AISC (Load and Resistance Factor Design-American Institute of Steel Constitution) [72], while the economy is observed in the overall or material cost of the frame. When formulated as a programming problem it turns out to be a discrete optimum design problem which has the following mathematical form:

$$
\begin{gathered}
\text { Minimize } \quad=\sum_{r=1}^{\mathrm{ng}} m_{r} \sum_{s=1}^{t_{r}} \ell_{s}, \\
\text { Subject to } \frac{\left(\delta_{j}-\delta_{j-1}\right)}{h_{j}} \leq \delta_{j u}, \quad j=1, \ldots, \mathrm{ns}, \\
\delta_{i} \leq \delta_{i u}, \quad i=1, \ldots, \mathrm{nd}, \\
\frac{P_{u k}}{\phi P_{n k}}+\frac{8}{9}\left(\frac{M_{u x k}}{\phi_{b} M_{n x k}}+\frac{M_{u y k}}{\phi_{b} M_{n y k}}\right) \leq 1, \\
\frac{P_{u k}}{2 \phi P_{n k}}+\frac{8}{9}\left(\frac{M_{u x k}}{\phi_{b} M_{n x k}}+\frac{M_{u y k}}{\phi_{b} M_{n y k}}\right) \leq 1, \\
P_{n k} \\
m_{s} \leq m_{s-1}, \quad \text { for } \frac{P_{u k}}{\phi P_{n k}}<0.2, \\
B_{s c} \leq B_{s b}, \quad s=1, \ldots, \text { nu, } \\
\leq \phi_{s-1}, \quad, n b,
\end{gathered}
$$

where (55a) defines the weight of the frame, $m_{r}$ is the unit weight of the steel section selected from the standard steel sections table that is to be adopted for group $r, t_{r}$ is the total number of members in group $r$, and ng is the total number of groups in the frame. $l_{s}$ is the length of the member $s$ which belongs to the group $r$.

Inequality (55b) represents the interstorey drift of the multistorey frame. $\delta_{j}$ and $\delta_{j-1}$ are lateral deflections of two adjacent storey levels and $h_{j}$ is the storey height. ns is the total number of storeys in the frame. Equation (55c) defines the displacement restrictions that may be required to include other-than-drift constraints such as deflections in beams. nd is the total number of restricted displacements in the frame. $\delta_{j u}$ is the allowable lateral displacement. LRFD-AISC limits the horizontal deflection of columns due to unfactored imposed load and wind loads to height of column/400 in each storey of a building with more than one storey. $\delta_{i u}$ is the upper bound on the deflection of beams which is given as span/360 if they carry plaster or other brittle finish.

Inequalities (55d) and (55e) represent strength constraints for doubly and singly symmetric steel members subjected to axial force and bending. If the axial force in member $k$ is tensile force the terms in these equations are given as the following: $P_{u k}$ is the required axial tensile strength, $P_{n k}$ is the nominal tensile strength, $\phi$ becomes $\phi_{t}$ in the case of tension and called strength reduction factor which is given as 0.90 for yielding in the gross section and 0.75 for fracture in the net section, $\phi_{b}$ is the strength reduction 
factor for flexure given as $0.90, M_{u x k}$ and $M_{u y k}$ are the required flexural strength, $M_{n x k}$ and $M_{n y k}$ are the nominal flexural strength about major and minor axis of member $k$, respectively. It should be pointed out that required flexural bending moment should include second-order effects. LRFD suggests an approximate procedure for computation of such effects which is explained in C1 of LRFD. In this case the axial force in member $k$ is a compressive force, and the terms in inequalities (55d) and (55e) are defined as the following: $P_{u k}$ is the required compressive strength, $P_{n k}$ is the nominal compressive strength, and $\phi$ becomes $\phi_{c}$ which is the resistance factor for compression given as 0.85 . The remaining notations in inequalities (55d) and (55e) are the same as the definition given previously.

The nominal tensile strength of member $k$ for yielding in the gross section is computed as $P_{n k}=F_{y} A_{g k}$ where $F_{y}$ is the specified yield stress and $A_{g k}$ is the gross area of member $k$. The nominal compressive strength of member $k$ is computed as $P_{n k}=A_{g k} F_{\text {cr }}$ where $F_{\text {cr }}=\left(0.658^{\lambda_{c}^{2}}\right) F_{y}$ for $\lambda_{c} \leq 1.5$ and $F_{\text {cr }}=\left(0.877 / \lambda_{c}^{2}\right) F_{y}$ for $\lambda_{c}>1.5$ and $\lambda_{c}=(K l / r \pi) \sqrt{F_{y} / E}$. In these expressions $E$ is the modulus of elasticity, and $K$ and $l$ are the effective length factor and the laterally unbraced length of member, $k$ respectively.

Inequality (55f) represents the strength requirements for beams in load and resistance factor design according to LRFD-F2. $M_{u x t}$ and $M_{n x t}$ are the required and the nominal moments about major axis in beam $b$, respectively. $\phi_{b}$ is the resistance factor for flexure given as $0.90 . M_{n x t}$ is equal to $M_{p}$, plastic moment strength of beam $b$ which is computed as $Z F_{y}$ where $Z$ is the plastic modulus and $F_{y}$ is the specified minimum yield stress for laterally supported beams with compact sections. The computation of $M_{n x b}$ for noncompact and partially compact sections is given in Appendix $F$ of LRFD. Inequality (55f) is required to be imposed for each beam in the frame to ensure that each beam has the adequate moment capacity to resist the applied moment. It is assumed that slabs in the building provide sufficient lateral restraint for the beams.

Inequality $(55 \mathrm{~g})$ is included in the design problem to ensure that the flange width of the beam section at each beamcolumn connection of storey should be less than or equal to the flange width of column section.

Inequalities (55h) and (55i) are required to be included to make sure that the depth and the mass per meter of column section at storey $s$ at each beam-column connection are less than or equal to width and mass of the column section at the lower storey $s-1$. nu is the total number of these constraints.

The solution of the optimum design problems described previously requires the selection of appropriate steel sections from a standard list such that the weight of the frame becomes the minimum while the constraints are satisfied. This turns the design problem into a discrete programming problem. As mentioned earlier the solution techniques available among the mathematical programming methods for obtaining the solution of such problems are somewhat cumbersome and not very efficient for practical use. Consequently structural optimization has not enjoyed the same popularity among the practicing engineers as the one it has enjoyed among the researchers. On the other hand, the emergence of new computational techniques called as stochastic search techniques or metaheuristic optimization algorithms that are based on the simulation of paradigms found in nature has changed this situation altogether. These techniques are inspired by analogies with physics, with biology, or with ethology.

\section{Stochastic Solution Techniques for Steel Frame Design Optimization Problems}

The stochastic search techniques make use of ideas inspired from nature, and they are equally efficient for obtaining the solution of both continuous and discrete optimization problems. The basic idea behind these techniques is to simulate the natural phenomena such as survival of the fittest, immune system, swarm intelligence, and the cooling process of molten metals through annealing to a numerical algorithm [87-107]. These methods are nontraditional stochastic search and optimization methods, and they are very suitable and effective in finding the solution of combinatorial optimization problems. They do not require the gradient information or the convexity of the objective function and constraints, and they use probabilistic transition rules not deterministic rules. Furthermore, they do not even require an explicit relationship between the objective function and the constraints. Instead they are based stochastic search techniques that make them quite effective and versatile to counter the combinatorial explosion of the possibilities. An extensive and detailed review of the stochastic search techniques employed in developing optimum design algorithms for steel frames is given in [16]. This review covers the articles published in the literature until 2007. In this paper firstly the summary of stochastic search algorithms is given, and the relevant publications after 2007 are reviewed in detail.

5.1. Evolutionary Algorithms. Evolutionary algorithms are based on the Darwinian theory of evolution and the survival of the fittest. They set up an artificial population that consists of candidate solutions to optimum design problem which are called individuals. These individuals are obtained by collecting the randomly selected values from the discrete set for each design variable. For example in a design problem of a steel frame with three design variables, the 11 th, $23^{\text {rd }}$, and 41st steel sections in the standard section list that contains 64 sections can randomly be selected for each design variable. First these sequence numbers are expressed in binary form as 001011, 010111, and 101001. This is called encoding. There are different types of encodings such as binary, realnumber, integer, and literal permutation encodings. When these binary numbers are combined together an individual consisting of zeros and ones such as 001011010111101001 is obtained. This individual is inserted to the population as a candidate solution. The reason 6 digits are used in the binary representation is to allow the possibility of covering total 64 sections available in the standard section list. The number of individuals can be generated same way and collected together to set up an artificial population. The binary representation of the individual is called its genome or chromosome. Each 
genome consists of a sequence of genes. A value of a gene is called an allele or string. It is apparent that all these terms are taken from cellular biology. It can be noticed that a new individual can be obtained by just changing one allele. Evolutionary algorithms do exactly this. They modify the genomes in the population to obtain a new individual which are called offspring or a child. Each individual is then checked for its quality which indicates its fitness as a solution for the design problem under consideration. A new population which is called a new generation is then obtained by keeping many of those offsprings with high fitness value and letting the ones with low fitness value to die off. Populations are generated one after another until one individual dominates either certain percentage of the population or after a predetermined number of generations. The individual with the highest fitness value is considered the optimum solution in both approaches. An extensive surveys of evolutionary computation and structural design are carried out in [90, 108-111].

There are varieties of evolutionary algorithms though in general they all are population-based stochastic search algorithms. They differ in the production of the new generations. However, those that are used in steel frame optimization can be collected under two main titles. These are genetic algorithms developed by Holland [87] and evolution strategies developed by Goldberg and Santani [112], Gen and Chang [113], and Chambers [98].

5.1.1. Genetic Algorithms. Genetic algorithm makes use of three basic operators to generate a new population from the current one $[16,90,114]$. The first one is known as selection, which involves selection of the individuals from the current population for mating depending on their fitness value. For each individuals a fitness value is calculated which represents the suitability of the individual's potential to be selected as a solution for the design. More highly fit designs send more copies to the mating pool. The fitness of individuals is calculated from the fitness criteria. In order to establish fitness criteria it is necessary to transform the constrained design problem into an unconstrained one. This is achieved by using a penalty function that generates a penalty to the objective function, whenever the constraints are violated. There are various forms of penalty functions used in conjunction with genetic algorithms. The details of these transformations are given in $[98,113,115]$.

The second operator is called crossover in which the strings of parents selected from the mating pool are broken into segments, and some of these segments are exchanged with corresponding segments of the other parent's string. The crossover operator swaps the genetic information between the mating pair of individuals. There are many different strategies to implement crossover operator such as fixed, flexible, and uniform crossovers [108, 115].

After the application of crossover new individuals are generated with different strings. These constitute the new population. Before repeating the reproduction and crossover once more to obtain another population the third operator called mutation is used. Mutation safeguards the process from a complete premature loss of valuable genetic material during reproduction and crossover. To apply mutation, few individuals of the population are randomly selected, and the string of each individual at a random location if 0 is switched to 1 or vice versa. The mutation operation can be beneficial in reintroducing diversity in a population.

Although initially genetic algorithms used the binary alphabet to code the search space solutions, there are also applications where other types of coding are utilized. Real coding seems particularly natural when tackling optimization problems of parameters with variables in continuous domains [116]. Leite and Topping [117] proposed modifications to one-point crossover by defining effective crossover site and using multiple off-spring tournaments. Modifications also covered to improve the efficiency of genetic operators to allow reduction in computation time and improve the results. Genetic algorithm has been extensively used in discrete optimization design of steel-framed structures [118-133].

Camp et al. [124, 125] developed a method for the optimum design of rigid plane skeleton structures subjected to multiple loading cases using genetic algorithm. The design constraints were implemented according to Allowable Stress Design specifications of American Institutes of Steels Construction (AISC-ASD). The steel sections were selected from AISC database of available structural steel members. Several strategies for reproduction and crossover were investigated. Different penalty functions and fitness policies were used to determine their suitability to ASD design formulation. Saka and Kameshki [126] presented genetic algorithm based algorithm design of multistory steel frames with sideway subjected to multiple loading cases. The design constraints that include serviceability as well as the combined strength constraints were implemented from British Standards BS5950. Saka [127] used genetic algorithm in the minimum design of grillage systems subjected to deflection limitations and allowable stress constraints. Weldali and Saka [128] applied the genetic algorithm to the optimum geometry and spacing design of roof trusses subjected to multiple loading cases. The algorithm obtains a roof truss that has the minimum weight by selecting appropriate steel sections from the standard British Steel Sections tables while satisfying the design limitations specified by BS5950. Saka et al. [129] presented genetic algorithm based optimum design method that find the optimum spacing in addition to optimum sections for the grillage systems. Deflection limitations and allowable stress constraints were considered in the formulation of the design problem. Pezeshk et al. [130] also presented a genetic algorithm based optimization procedure for the design of plane frames including geometric nonlinearity. The design constraints are accounted for AISCLRFD specifications. Hasançebi and Erbatur [131] carried out evaluation of crossover techniques in genetic algorithm. For this purpose commonly used single-point, 2-point, multipoint, variable-to-variables and uniform crossover are used in the optimum design of steel pin jointed frames. They have concluded that two-point crossover performed better than other crossover types. Erbatur et al. [132] developed GAOS program which implements the constraints from the design code and determines the optimum readymade hot-rolled 
sections for the steel trusses and frames. Kameshki and Saka [133] used genetic algorithm to compare the optimum design of multistory nonswaying steel frames with different types of bracing. Kameshki and Saka [134] presented an application of the genetic algorithm to optimum design of semirigid steel frames. The design algorithm considers the service ability and strength constraints as specified in BS5950. Andre et al. [135] discussed the trade-off between accuracy, reliability, and computing time in the binary-encoded genetic algorithm used for global optimization over continuous variables. Large set of analytical test functions are considered to test the effectiveness of the genetic algorithm. Some improvements such as Gray coding and double crossover are suggested for a better performance. Toropov and Mahfouz [136] presented genetic algorithm based structural optimization technique for the optimum design of steel frames. Certain modifications are suggested which improved the performance of the genetic algorithm. The algorithm implements the design constraints from BS5950 and considers the wind loading from BS6399. The steel sections are selected from BS4. Hayalioğlu [137] developed a genetic algorithm based optimum design algorithm for three-dimensional steel frames which implements the design constraints from LRFD-AISC. The wind loading is taken from Uniform Building Code (UBC). The algorithm is also extended to include the design constraints according to Allowable Stress Design (ASD) of AISC for comparison. Jenkins [138] used decimal coding instead of binary coding in genetic algorithm. The performance of the decimal coded genetic algorithm is assessed in the optimum design of 640bar space deck. It is concluded that decimal coding avoids the inevitable large shifts in decoded values of variables when mutation operates at the significant end of binary string. Kameshki and Saka [139] extended the work of [134] to frames with various semirigid beam-to-column connections such as end plate without column stiffeners, top and seat angle with double web angle, top and seat angle without double web angle. Yun and Kim [140] presented optimum design method for inelastic steel frames. Kaveh and Shahrouzi [141] utilized a direct index coding within the genetic algorithm, such a way that the optimization algorithm can simultaneously integrate topology and size in a minimal length chromosome. Balling et al. [142] also presented a genetic algorithm based optimum design approach for steel frames that can simultaneously optimize size, shape, and topology. It finds multiple optimums and near optimum topologies in a single run. Toğan and Daloğlu [143] suggested the adaptive approach in genetic algorithm which eliminates the necessity of specifying values for penalty parameter and probabilities of crossover and mutation. Kameshki and Saka [144] presented an algorithm for the optimum geometry design of geometrically nonlinear geodesic domes that uses genetic algorithm and elastic instability analysis.

Degertekin [145] compared the performance of the genetic algorithm with simulated annealing in the optimum design of geometrically nonlinear steel space frames where the design formulation is carried out considering both LRFD and ASD specifications of AISC. It is concluded that simulated annealing yielded better designs. Later in [146] the performance of genetic algorithm is compared with that of tabu search in finding the optimum design of steel space frames and found that tabu search resulted in lighter frames. Issa and Mohammad [147] attempted to modify a distributed genetic algorithm to minimize the weight of steel frames. They have used twin analogy and a number of mutation schemes and reported improved performance for genetic algorithm. Safari et al. [148] have proposed new crossover and mutation operators to improve the performance of the genetic algorithm for the optimum design of steel frames. Significant improvements in the optimum solutions of the design examples considered are reported.

5.1.2. Evolution Strategies. Evolution strategies were originally developed for continuous structural optimization problems [149]. These algorithms are extended to cover discrete design problems by Cai and Thierauf [150]. The basic differences between discrete and continuous evolution strategies are in the mutation and recombination operators. Evolution strategies algorithm randomly generates an initial population consisting of $\mu$ parent individuals. It then uses recombination, mutation, and selection operators to attain a new population. The steps of the method are as follows $[16,149]$.

(1) Recombination. The population of $\mu$ parents is recombined to produce $\lambda$ off springs in $i$ th generation in order to allow the exchange of design characteristics on both levels of design variables and strategy parameters. For every offspring vector a temporary parent vector $s=\left\{s_{1}, s_{2}, \ldots, s_{n}\right\}$ is first built by means of recombination. The following operators can be used to obtain the recombined $s^{\prime}$ :

$$
s_{i}^{\prime}= \begin{cases}s_{a, i} & \text { no recombination, } \\ s_{a, i} \text { or } s_{b, i} & \text { discrete, } \\ s_{a, i} \text { or } s_{b j, i} & \text { global discrete, } \\ s_{a, i}+\frac{\left(s_{b, i}-s_{a, i}\right)}{2} & \text { intermediate } \\ s_{a, i}+\frac{\left(s_{b j, i}-s_{a, i}\right)}{2} & \text { global intermediate }\end{cases}
$$

where $s_{a}$ and $s_{b}$ refer to the $s$ component of two parent individuals which are randomly chosen from the parent population. First case corresponds to no recombination case in which $s^{\prime}$ is directly formed by copying each element of first parent $s_{a, i}$. In the second, $s^{\prime}$ is chosen from one of the parents under equal probability. In the third, the first parent is kept unchanged, while a new second parent $s_{b j}$ is chosen randomly from the parent population. The fourth and fifth cases are similar to second and third cases, respectively; however, arithmetic means of the elements are calculated.

(2) Mutation. This operator is not only applied to design variables but also strategy parameters. Carrying out the mutation of strategy parameters first and the design variables later increases the effectiveness of the algorithm. It is suggested in [149] that not all of the components of the parent individuals but only a 
few of them should randomly be changed in every generation.

(3) Selection. There are two types of selection application. In the first the best $\mu$ individuals are selected from a temporary population of $(\mu+\lambda)$, individuals to form the parents of the next generation. In the second the $\mu$ individuals produce $\lambda$ off springs $(\mu \leq \lambda)$ and the selection process defines a new population of $\mu$ individuals from the set of $\lambda$ off springs only.

(4) Termination. The discrete optimization procedure is terminated either when the best value of the objective function in the last $4 n \mu / \lambda$ or when the mean value of the objective values from all parent individuals in the last $4 n \mu / \lambda$ generations has not been improved by less than a predetermined value $\varepsilon$.

There are different types of constraint handling in evolution strategies method. An excellent review of these is given in [151]. In nonadaptive evolution strategies constraint handling is such that if the individual represents an infeasible design point it is discarded from the design space. Hence only the feasible individuals are kept in the population. For this reason many potential designs that are close to acceptable design space are rejected that results in a loss of valuable information. The adaptive evolution strategies suggested in [152] use soft constraints during the initial stages of the search; the constraints become severe as the search approaches the global optimum. The detailed steps of this algorithm are given in [149] where the procedure is explained in a simple example of three-bar steel truss structure. Later, two steel space frames are designed with evolution strategies in which the effect of different selection schemes and constraint handling is studied.

Rajasekaran et al. [153] applied the evolutionary strategies method to the optimum design of large-size steel space structures such as a double-layer grid with 700 degrees of freedom. It is reported that evolutionary strategies method worked effectively to obtain the optimum solutions of these largesize structures. Later Rajasekaran [154] used the same method to obtain the optimum design of laminated nonprismatic thin-walled composite spatial members that are subjected to deflection, buckling, and frequency constraints. Evolutionary strategies technique is applied to determine the optimal fibre orientation of composite laminates.

Ebenau et al. [155] combined evolutionary strategies technique with an adaptive penalty function and a special selection scheme. The algorithm developed is applied to determine the optimum design of three-dimensional geometrically nonlinear steel structures where the design variables were mixed, discrete, or topological.

Hasançebi [156] has compared three different reformulations of evolution strategies for solving discrete optimum design of steel frames. Extensive numerical experimentation is performed in the design examples to facilitate a statistical analysis of their convergence characteristics. The results obtained are presented in the histograms demonstrating the distribution of the best designs located by each approach. Further in [157], the same author investigated the application of evolution strategies to optimize the design of a truss bridge.
The design problem involved identifying the optimum topology, shape, and member sizes of the bridge. The design problem consisted of mixed continuous and discrete design variables. It is shown that evolutionary strategies efficiently found the optimum topology configuration of a bridge in a large and flexible design space.

Hasançebi [158] has improved the computational performance of evolution strategies algorithm by suggesting selfadaptive scheme for continuous and discrete steel frame design problems. A numerical example taken from the literature is studied in depth to verify the enhanced performance of the algorithm, as well as to scrutinize the role and significance of this self-adaptation scheme. It is shown that adaptive evolution strategies algorithms are reliable and powerful tools and well-suited for optimum design of complex structural systems, including large-scale structural optimization.

5.2. Simulated Annealing. Simulated annealing is an iterative search technique inspired by annealing process of metals. During the annealing process a metal first is heated up to high temperatures until it melts which imparts high energy to it. In this stage all molecules of the molten metal can move freely with respect to each other. The metal is then cooled slowly in a controlled manner such that at each stage a temperature is kept of sufficient duration. The atoms then arrange themselves in a low-energy state and leads to a crystallized state which is a stable state that corresponds to an absolute minimum of energy. On the other hand if the cooling is carried out quickly the metal forms polycrystalline state which corresponds to a local minimum energy. The metal reaches thermal equilibrium at each temperature level $T$ described by a probability of being in a state $i$ with energy $E_{i}$ given by the Boltzman distribution:

$$
P_{r}=\frac{1}{Z(T)} \exp \left(\frac{-E_{i}}{k_{B} T}\right),
$$

where $Z(T)$ is a normalization factor and $k_{B}$ is Boltzmann constant. The Boltzmann distribution focuses on the states with the lowest energy as the temperature decreases. An analogy between the annealing and the optimization can be established by considering the energy of the metal as the objective function, while the different states during the cooling represent the different optimum solutions (designs) throughout the optimization process. In the application of the method first the constrained design problem is transferred into an unconstrained problem by using penalty function. If the value of the unconstrained objective function of the randomly selected design is less than the one in the current design, then the current design is replaced by the new design. Otherwise the fate of the new design is decided according to the Metropolis algorithm as explained in the following:

$$
p_{i j}\left(T_{k}\right)= \begin{cases}1 & \text { if } \Delta W_{i j} \leq 0, \\ \exp \left(\frac{-\Delta W_{i j}}{\Delta W T_{k}}\right) & \text { if } \Delta W_{i j}>0,\end{cases}
$$

where $p_{i j}$ is the acceptance probability of selected design. $\Delta W_{i j}=W_{j}-W_{i}$ in which $W_{j}$ and $W_{i}$ are the objective 
function values of the selected and current designs, $\Delta W$ is a normalization constant which is the running average of $\Delta W_{i j}$, and $T_{k}$ is the strategy temperature. $\Delta W$ is updated when $\Delta W_{i j}>0$ as explained in [88].

The strategy temperature $T_{k}$ is gradually decreased, while the annealing process proceeds according to a cooling schedule. For this the initial and the final values of the temperature $T_{s}$ and $T_{f}$ are to be known. Once the starting acceptance probability $P_{s}$ is decided the starting temperature is calculated from $T_{s}=-1 / \ln \left(P_{s}\right)$. The strategy temperature is then reduced using $T_{k+1}=\alpha T_{k}$ where $\alpha$ is the cooling factor and is less than one. While $T$ approaches zero, $p_{i j}$ also approaches zero. For a given final acceptance probability, the final temperature is calculated from $T_{f}=-1 / \ln \left(P_{f}\right)$. After $N$ cycles the final temperature value $T_{f}$ can be expressed as $T_{f}=T_{s} \alpha^{N-1}$.

Simulated annealing is originated by Kirkpatrick et al. [88] and Černý [159] independently at the same time which is based on the previously mentioned phenomenon. The steps of the optimum design algorithm for steel frames using simulated annealing method are given in the following. The method is based on the work of Bennage and Dhingra [160], and the normalization constant in the Metropolis algorithm [161] is taken from the work of Balling [162]. The details of these steps are given in $[16,145]$.

(1) Select the values for $P_{s}, P_{f}$, and $N$ and calculate the cooling schedule parameters $T_{s}, T_{f}$, and $\alpha$ as explained previously. Initialize the cycle counter $i c=$ 1.

(2) Generate an initial design randomly where each design variable represents the sequence number of the steel section randomly selected from the list. The initial design is assigned as current design. Carry out the analysis of the frame with steel section selected in the current design and obtain its response under the applied loads. Calculate the value of objective function $W$.

(3) Determine the number of iterations required per cycle from the equation mentioned later.

$$
i=i_{f}+\left(i_{f}-i_{s}\right)\left(\frac{T-T_{f}}{T_{f}-T_{s}}\right),
$$

where $i_{s}$ and $i_{f}$ are the number of iterations per cycle required at the initial and final temperatures $T_{s}$ and $T_{f}$.

(4) Select a variable randomly. Apply a random perturbation to this variable, and generate a candidate design in the neighbourhood of the current design. Compute $\Delta W_{i j}$.

(5) Accept this candidate design as the current design if $\Delta W_{i j} \leq 0$.

(6) If $\Delta W_{i j}>0$ then update $\Delta W$. Calculate the acceptance probability $p_{i j}$ from (11). Generate a uniformly distributed random number $r$ over interval $[0,1]$. If $r<p_{i j}$ go to next step otherwise go to step 4 .
(7) Accept the candidate design as the current design. If the current design is feasible and is better than the previous optimum design, assign it temporarily as the optimum design.

(8) Update the temperature $T_{k}$ using $T_{k+1}=\alpha T_{k}$. Increase the cycle counter by one; $i c=i c+1$. If $i c>N$ terminate the algorithm and take the last temporary optimum as the final optimum design. Otherwise return to step 3 .

Balling [162] adapted simulated annealing strategy for the discrete optimum design of three-dimensional steel frames. The total frame weight is minimized subject to designcode-specified constraints on stress, buckling, and deflection. Three loading cases are considered. In the first loading case a uniform live load was placed on all floors and the roof. In the second and third loading cases horizontal seismic loads in the global $X$ and $Z$ directions were placed at various nodes, respectively, according to Uniform Building Code. Members in the frame were selected from among the discrete standardized shapes. Some approximation techniques were used to reduce the computation time. Later in [163] a filter is suggested through which each design candidate must pass before it is accepted for computationally intensive structural analysis is set-up. A scheme is devised whereby even less feasible candidates can pass through the filter in a probabilistic manner. It is shown that the filter size can speed up convergence to global optimum.

Simulated annealing is applied to optimum design of large tetrahedral truss for minimum surface distortion in $[164,165]$. In [166], the simulated annealing is applied to large truss structures where the standard cooling schedule is modified such that the best solution found so far is used as a starting configuration each time the temperature is reduced.

Topping et al. [167] used simulated annealing in simultaneous optimum design for topology and size. The algorithm developed is applied to truss examples from the literature for which the optimum solutions were known. The effects of control parameters are discussed. Later in [168] implementation of parallel simulated annealing models is evaluated by the same authors. It is stated in this work that efficiency of parallel simulated annealing is problem dependant and some engineering problems solution spaces may be very complex and highly constrained. Study provides guidelines for the selection of appropriate schemes in such problems. Tzan and Pantelides [169] developed an annealing method for optimal structural design with sensitivity analysis and automatic reduction of the search range.

Hasançebi and Erbatur [170] presented simulated annealing based structural optimization algorithm for large and complex steel frames. Two general and complementary approaches with alternative methodologies to reformulate the working mechanism of the Boltzmann parameter are introduced to accelerate the convergence reliability of simulated annealing. Later in [171] they have developed simulated annealing based algorithm for the simultaneous optimum design of pin-jointed structures where the size, shape, and topology are taken as design variables. In the examples considered while the weight of trusses is minimized the design 
constraints such as nodal displacements, member stress, and stability are implemented from design code specifications.

Degertekin [172] proposed a hybrid tabu-simulated annealing algorithm for the optimum design of steel frames. The algorithm exploits both tabu search and simulated annealing algorithms simultaneously to obtain near optimum solution. The design constraints are implemented from LRDAISC specification. It is reported that hybrid algorithm yielded lighter optimum structures than those algorithms considered in the study.

Hasancebi et al. [173] have suggested novel approaches to improve the performance of simulated annealing search technique in the optimum design of real-size steel frames to eliminate its drawbacks mentioned in the literature. It is reported that the suggested improvements eliminated the drawbacks in the design examples considered in the study. In [174] the same author has used simulated annealing based optimum design method to evaluate the topological forms for single span steel truss bridge. The optimum design algorithm attains optimum steel profiles for the members and the truss as well as optimum coordinates of top chord joints so that the bridge has the minimum weight. The design constraints and limitations are imposed according to serviceability and strength provisions of ASD-AISC (Allowable Stress Design Code of American Institute of Steel Institution) specification.

5.3. Particle Swarm Optimizer. Particle swarm optimizer is based on the social behavior of animals such as fish schooling, insect swarming, and birds flocking. This behavior is concerned with grouping by social forces that depend on both the memory of each individual as well as the knowledge gained by the swarm $[175,176]$. The procedure involves a number of particles which represent the swarm and are initialized randomly in the search space of an objective function. Each particle in the swarm represents a candidate solution of the optimum design problem. The particles fly through the search space, and their positions are updated using the current position, a velocity vector, and a time increment. The steps of the algorithm are outlined in the following as given in $[177,178]$.

(1) Initialize swarm of particles with positions $x_{0}^{i}$ and initial velocities $v_{0}^{i}$ randomly distributed throughout the design space. These are obtained from the following expressions:

$$
\begin{gathered}
x_{0}^{i}=x_{\min }+r\left(x_{\max }-x_{\min }\right) . \\
v_{0}^{i}=\left[\frac{\left(x_{\min }+r\left(x_{\max }-x_{\min }\right)\right)}{\Delta t}\right],
\end{gathered}
$$

where the term $r$ represents a random number between 0 and 1 , and $x_{\min }$ and $x_{\max }$ represent the design variables of upper and lower bounds, respectively.

(2) Evaluate the objective function values $f\left(x_{k}^{i}\right)$ using the design space positions $x_{k}^{i}$.
(3) Update the optimum particle position $p_{k}^{i}$ at the current iteration $k$ and the global optimum particle position $p_{k}^{g}$.

(4) Update the position of each particle from $x_{k+1}^{i}=$ $x_{k}^{i}+v_{k+1}^{i} \Delta t$ where $x_{k+1}^{i}$ is the position of particle $i$ at iteration $k+1, v_{k+1}^{i}$ is the corresponding velocity vector, and $\Delta t$ is the time step value.

(5) Update the velocity vector of each particle. There are several formulas for this depending on the particular particle swarm optimizer under consideration. The one given in $[176,177]$ has the following form:

$$
v_{k+1}^{i}=w v_{k}^{i}+c_{1} r_{1} \frac{\left(p_{k}^{i}-x_{k}^{i}\right)}{\Delta t}+c_{2} r_{2} \frac{\left(p_{k}^{g}-x_{k}^{i}\right)}{\Delta t},
$$

where $r_{1}$ and $r_{2}$ are random numbers between 0 and $1, p_{k}^{i}$ is the best position found by particle $i$ so far, and $p_{k}^{g}$ is the best position in the swarm at time $k$. $w$ is the inertia of the particle which controls the exploration properties of the algorithm. $c_{1}$ and $c_{2}$ are trust parameters that indicate how much confidence the particle has in itself and in the swarm, respectively.

(6) Repeat steps 2-5 until stopping criteria are met.

Fourie and Groenwold [178] applied particle swarm optimizer algorithm to optimal design of structures with sizing and shape variables. Standard size and shape design problems selected from the literature are used to evaluate the performance of the algorithm developed. The performance of particle swarm optimizer is compared with three-gradient based methods and genetic algorithm. It is reported that particle swarm optimizer performed better than genetic algorithm.

Perez and Behdinan [179] presented particle swarm based optimum design algorithm for pin jointed steel frames. Effect of different setting parameters and further improvements are studied. Effectiveness of the approach is tested by considering three benchmark trusses from the literature. It is reported that the proposed algorithm found better optimal solutions than other optimum design techniques considered in these design problems.

In [180] particle swarm optimizer is improved by introducing fly-back mechanism in order to maintain a feasible population. Furthermore, the proposed algorithm is extended to handle mixed variables using a simple scheme and used to determine the solution of five benchmark problems from the literature that are solved with different optimization techniques. It is reported that the proposed algorithm performed better than other techniques. In [181] particle swarm optimizer is improved by considering a passive congregation which is an important biological force preserving swarm integrity. Passive congregation is an attraction of an individual to other group members, but where there is no display of social behavior. Numerical experimentation of the algorithm is carried out on 10 benchmark problems taken from the literature, and it is stated that this improvement enhances the search performance of the algorithm significantly. 
Li et al. [182] presented a heuristic particle swarm optimizer for the optimum design of pin jointed steel frames. The algorithm is based on the particle swarm optimizer with passive congregation and harmony search scheme. The method is applied to optimum design of five-planar and spatial truss structures. The results show that proposed improvements accelerate the convergence rate and reache to optimum design quicker than other methods considered in the study.

Doğan and Saka [183] presented particle swarm based optimum design algorithm for unbraced steel frames. The design constraints imposed are in accordance with LRFDAISC code. The design algorithm selects optimum W sections for beams and columns of unbraced steel frames such that the design constraints are satisfied and the minimum frame weight is obtained.

5.4. Ant Colony Optimization. Ant colony optimization technique is inspired from the way that ant colonies find the shortest route between the food source and their nest. The biologists studied extensively for a long time how ants manage collectively to solve difficult problems in a natural way which is too complex for a single individual. Ants being completely blind individuals can successfully discover as a colony the shortest path between their nest and the food source. They manage this through their typical characteristic of employing volatile substance called pheromones. They perceive these substances through very sensitive receivers located in their antennas. The ants deposit pheromones on the ground when they travel which is used as a trail by other ants in the colony. When there is a choice of selection for an ant between two paths it selects the one where the concentration of pheromone is more. Since the shorter trail will be reinforced more than the long one after a while a great majority of ants in the colony will travel on this route. Ant colony optimization algorithm is developed by Colorni et al. [184] and Dorigo [185, 186] and used in the solution of travelling salesman. The steps of optimum design algorithm for steel frames based on ant colony optimization are as follows $[187,188]$.

(1) Calculate an initial trail value as $\tau_{0}=1 / w_{\min }$ where $w_{\min }$ is the weight of the frame from assigning the smallest steel sections from the database to each member group of the frame.

(2) Assign a member group to each ant in the colony randomly, and then select a steel section from the database so that the ant can start its tour. This selection is carried out according to the following decision process:

$$
a_{i j}(t)=\frac{\left[\tau_{i j}(t)\right]\left[v_{i j}\right]^{\beta}}{\sum_{k=1}^{n s_{i}}\left[\tau_{i k}(t)\right]\left[v_{i k}\right]^{\beta}},
$$

where $j$ is the steel section assigned to member group $i$, and $n s_{i}$ is the total number of steel sections in the database. $\beta$ is a constant. The probability $p_{i j}^{\ell}(t)$ that the ant $\ell$ assigns a steel section $j$ to member group $i$ at time $t$ is given as

$$
p_{i j}^{\ell}(t)=\frac{a_{i j}(t)}{\sum_{k=1}^{n s_{i}} a_{i \ell}(t)} .
$$

(3) After the steel section is selected by the first ant as explained in step 2, the intensity of trail on this path is lowered using local update rule $\tau_{i j}(t)=\xi \tau_{i j}(t)$ where $\zeta$ is an adjustable parameter between 0 and 1 .

(4) The second ant selects a steel section from the database for its member group $i$ and a local update rule is applied. This procedure is continued until all the ants in the colony select a steel section for the starting member group in their position on the frame. After completing the first iteration of the tour, each ant selects another steel section to its next member group $i+1$. This procedure is repeated until each ant in the colony has selected a steel section from the database for each member group in the frame. Hence when the selection process is completed the ant colony has $m$ different designs for the frame where $m$ represents the total number of ants selected initially.

(5) Frame is analyzed for these designs and the violations of constraints corresponding to each ant are calculated and substituted into the penalty function of $F=$ $W(1+C)^{\alpha}$ where $W$ is the weight of the frame, $C$ is the total constraint violation, and $\alpha$ is the penalty function exponent. All designs are in a cycle and are ranked by their penalized weights, and the elitist ant that selected the frame with the smallest penalized weight in all cycles is determined. The amount of trail $\Delta \tau_{i j}^{e}=1 / W_{e}$ is added to each path chosen by this elitist ant where $W_{e}$ is the penalized frame weight selected by the elitist ant.

(6) Carry out the global update of trails from $\tau_{i j}(t+1)=$ $\rho \tau_{i j}(t)+(1-\rho) \Delta \tau_{i j}$ where $\rho$ is a constant having value between 0 and 1 that represents the evaporation rate and $\Delta \tau_{i j}=\sum_{k=1}^{m} \Delta \tau_{i j}^{k}$ in which $m$ is the total number of ants selected initially.

Camp and Bichon [187] developed discrete optimum design procedure for space trusses based on ant colony optimization technique. The total weight of the structure is minimized, while stress and deflection limitations are considered. The design problem is transformed into modified travelling salesman problem where the network of travelling salesman is taken as the structural topology and the length of the tour is the weight of the structure. The number of trusses is designed using the algorithm developed and results obtained are compared with genetic algorithm and gradient based optimization methods. Later in [188] the work is extended to rigid steel frames. The serviceability and strength constraints are implemented from LRFD-AISC-2001 code. A comparison is presented between ant colony optimization frame design and designs obtained using genetic algorithm.

Kaveh and Shojaee [189] also used ant colony optimization algorithm to develop an approach for the discrete 
optimum design of steel frames. The design constraints considered consist of combined bending and compression, combined bending and tension, and deflection limitations which are specified according to ASD-AISC design code. The detailed explanation of ant colony optimization operators is given. Optimum designs of six plane steel structures are obtained using the algorithm developed. Some of the results are compared to those that are achieved by genetic algorithms which are taken from the literature. Bland [190] also used ant colony optimization to obtain the minimum weight of transmission tower which has over 200 members. Kaveh and Talatahari [191] presented an improved ant colony optimization algorithm for the design of steel frames. The algorithm employs suboptimization mechanism based on the principals of finite element method to reduce the search domain, the size of trail matrix, and the number of structural analyses in the global phase, and the optimum design is obtained by considering $\mathrm{W}$ sections list in the neighborhood of the result attained in the previous phase. Wang et al. [192] presented an algorithm for a partial topology and member size optimization for wing configuration. Ant colony optimization is used to determine the optimum topology of the wing structure, and gradient based optimization method is used for component size optimization problem. It is stated that this combined procedure was effective in solving wing structure optimization problem. In [193] ant colony algorithm is used to develop design optimization technique for three-dimensional steel frames where the effect of elemental warping is taken into account.

5.5. Harmony Search Method. One other recent addition to metaheuristic algorithms is the harmony search method originated by Geem et al. [194-206]. Harmony search algorithm is based on natural musical performance processes that occur when a musician searches for a better state of harmony. The resemblance for an example between jazz improvisation that seeks to find musically pleasing harmony and the optimization is that the optimum design process seeks to find the optimum solution as determined by the objective function. The pitch of each musical instrument determines the aesthetic quality just as the objective function value is determined by the set of values assigned to each decision variable.

Harmony search algorithm consists of five basic steps. The detailed explanation of these steps can be found in [196] which are summarized in the following.

(1) Initialize the harmony search parameters. A possible value range for each design variable of the optimum design problem is specified. A pool is constructed by collecting these values together from which the algorithm selects values for the design variables. Furthermore the number of solution vectors in harmony memory (HMS) that is the size of the harmony memory matrix, harmony considering rate (HMCR), pitch adjusting rate (PAR), and the maximum number of searches is also selected in this step.

(2) Initialize the harmony memory matrix (HM). Each row of harmony memory matrix contains the values of design variables which randomly selected feasible solutions from the design pool for that particular design variable. Hence, this matrix has $n$ columns where $n$ is the total number of design variables and HMS rows which is selected in the first step. HMS is similar to the total number of individuals in the population matrix of the genetic algorithm.

(3) Improvise a new harmony memory matrix. In generating a new harmony matrix the new value of the $i$ th design variable can be chosen from any discrete value within the range of $i$ th column of the harmony memory matrix with the probability of HMCR which varies between 0 and 1 . In other words, the new value of $x_{i}$ can be one of the discrete values of the vector $\left\{x_{i, 1}, x_{i, 2}, \ldots, x_{i, \mathrm{hms}}\right\}^{T}$ with the probability of HMCR. The same is applied to all other design variables. In the random selection, the new value of the $i$ th design variable can also be chosen randomly from the entire pool with the probability of 1 -HMCR. That is

$$
\begin{aligned}
& x_{i}^{\text {new }} \\
& =\left\{\begin{array}{l}
x_{i} \in\left\{x_{i, 1}, x_{i, 2}, \ldots, x_{i, \mathrm{hms}}\right\}^{T} \text { with probability HMCR } \\
x_{i} \in\left\{x_{1}, x_{2}, \ldots, x_{\mathrm{ns}}\right\}^{T} \text { with probability (1 - HMCR), }
\end{array}\right.
\end{aligned}
$$

where ns is the total number of values for the design variables in the pool. If the new value of the design variable is selected among those of harmony memory matrix, this value is then checked for whether it should be pitch-adjusted. This operation uses pitch adjustment parameter PAR that sets the rate of adjustment for the pitch chosen from the harmony memory matrix as follows:

$$
\begin{aligned}
& \text { Is } x_{i}^{\text {new }} \text { to be pitch-adjusted? } \\
& \qquad\left\{\begin{array}{l}
\text { Yes with probability of PAR } \\
\text { No with probability of }(1-\text { PAR })
\end{array}\right\} .
\end{aligned}
$$

Supposing that the new pitch adjustment decision for $x_{i}^{\text {new }}$ came out to be yes from the test and if the value selected for $x_{i}^{\text {new }}$ from the harmony memory is the $k$ th element in the general discrete set, then the neighboring value $k+1$ or $k-1$ is taken for new $x_{i}^{\text {new }}$. This operation prevents stagnation and improves the harmony memory for diversity with a greater change of reaching the global optimum.

(4) Update the harmony memory matrix. After selecting the new values for each design variable the objective function value is calculated for the new harmony vector. If this value is better than the worst harmony vector in the harmony matrix, it is then included in the matrix, while the worst one is taken out of the matrix. The harmony memory matrix is then sorted in descending order by the objective function value.

(5) Repeat steps 3 and 4 until the termination criteria is satisfied. 
Lee and Geem [196] presented harmony search algorithm based discrete optimum design technique for plane trusses. Eight different plane trusses are selected from the literature and optimized using the approach developed to demonstrate the efficiency and robustness of the harmony search algorithm. In all these examples the proposed algorithm has found the minimum weight that is lighter than those determined by other techniques. In [197], authors have extended the harmony search algorithm to deal with continuous engineering optimization problems. Various engineering design examples including mathematical function minimization and structural engineering optimization problems are considered to demonstrate the effectiveness of the algorithm. It is concluded that the results obtained indicated that the proposed approach is a powerful search and optimization technique that may yield better solutions to engineering problems than those obtained using current algorithms.

Saka [207] presented harmony search algorithm based optimum geometry design technique for single-layer geodesic domes. It treats the height of the crown as design variable in addition to the cross-sectional designations of members. A procedure is developed that calculates the joint coordinates automatically for a given height of the crown. The serviceability and strength requirements are considered in the design problem as specified in BS5950-2000. This code makes use of limit state design concept in which structures are designed by considering the limit states beyond which they would become unfit for their intended use. The design examples considered have shown that harmony search algorithm obtains the optimum height and sectional designations for members in relatively less number of searches. Later this technique is extended to cover the optimum topology design of nonlinear lamella and network domes [208-210] where geometric nonlinearity is also taken into account.

Saka and Erdal [211] used harmony search algorithm to develop a discrete optimum design method for grillage systems. The displacement and strength specifications are implemented from LRFD-AISC. The algorithm selects the appropriate $\mathrm{W}$ sections from the database for transverse and longitudinal beams of the grillage system. The number of design examples is considered to demonstrate the efficiency of the proposed algorithm.

Saka [212] presented harmony search algorithm based discrete optimum design method for rigid steel frames. The objective is taken as the minimum weight of the frame and the behavioral and performance limitations are imposed from BS5950. The list of Universal Beam and Universal Column sections of The British Code is considered for the frame members to be selected from. The combined strength constraints that are considered for beam columns take into account the effect of lateral torsional buckling. The effective length computations for columns are automated and decided by the algorithm itself depending upon the steel sections adopted for beams and columns within that design cycle. It is demonstrated that harmony search algorithm is quite effective and robust in finding the solution of minimum weight steel frame design. Degertekin [213] also presented harmony search method based discrete optimum design method for steel frame where the design constraints are implemented according to LRFD-AISC specifications. The effectiveness and robustness of harmony search algorithm, in comparison with genetic algorithm, simulated annealing and colony optimization based methods and were verified using three planar and two-space steel frames. The comparisons showed that the harmony search algorithm yielded lighter designs for the presented examples. Degertekin et al. [214] used harmony search method to develop an optimum design algorithm for geometrically nonlinear semirigid steel frames where the steel sections are selected from European wide flange steel sections (HE sections). It is stated that harmony search method efficiently obtained the optimum solution of complex design problem requiring relatively less computational time.

Hasançebi et al. [215, 216] developed adaptive harmony search method for optimum design of steel frames where two of the three main operators of classical harmony search methods, namely, harmony memory considering rate and pitch adjusting rate are adjusted automatically by the algorithm itself during the design iterations. The initial values selected for these parameters directly affect the performance of the algorithm. This novel technique eliminates problemdependent value selection of these parameters. It is shown that adaptive harmony search technique performs much better than the standard harmony search method in obtaining the optimum designs of real-size steel frames.

Erdal et al. [217] formulated design problem of cellular beams as an optimum design problem by treating the depth, the diameter, and the total number of holes as design variables. The design problem is formulated considering the limitations specified in The Steel Construction Institute Publication Number 100 which is consisted BS5950 parts 1 and 3. The solution of the design problem is determined by using the harmony search algorithm and particle swarm optimizers. In the design examples considered it is shown that both methods efficiently obtained the optimum Universal beam section to be selected in the production of the cellular beam subjected to general loading, the optimum hole diameters, and the optimum number of holes in the cellular beam such that the design limitations are all satisfied.

Saka et al. [218] have evaluated the recent enhancements suggested by several authors in order to improve the performance of the standard harmony search method. Among these are the improved harmony search method and global harmony search method by Mahdavi et al. [219, 220], adaptive harmony search method [215], improved harmony search method by Santos Coelho and de Andrade Bernert [221], and dynamic harmony search method by Saka et al. [218]. The optimum design problem of steel space frames is formulated according to the provisions of LRFD-AISC (Load and Resistance Factor Design-American Institute of Steel Corporation). Seven different structural optimization algorithms are developed, each of which is based on one of the previously mentioned versions of harmony search method. Three real-size space steel frames, one of which has 1860 members, are designed using each of these algorithms. The optimum designs obtained by these techniques are compared, and performance of each version is evaluated. It is stated 
that, among all, the adaptive and dynamic harmony search methods outperformed the others.

5.6. Big Bang Big Crunch Algorithm. Big Bang-Big Crunch algorithm is developed by Erol and Eksin [222] which is a population based algorithm similar to the genetic algorithm and the particle swarm optimizer. It consists of two phases as its name implies. First phase is the big bang in which the randomly generated candidate solutions are randomly distributed in the search space. In the second phase these points are contracted to a single representative point that is the weighted average of randomly distributed candidate solutions. First application of the algorithm in the optimum design of steel frames is carried out by Camp [223]. The steps of the method are listed later as it is given in [223].

(1) Decide an initial population size and generate initial population randomly. These candidate solutions are scattered in the design domain. This is called the big bang phase.

(2) Apply contraction operation. This operation takes the current position of each candidate solution in the population and its associated penalized fitness function value and computes a center of mass. The center of mass is the weighted average of the candidate solution positions with respect to the inverse of the penalized fitness function values:

$$
x_{\mathrm{cm}}=\left[\sum_{i=1}^{\mathrm{np}} \frac{x_{i}}{f_{i}}\right] \div\left[\sum_{i=1}^{n} \frac{1}{f_{i}}\right] \text {, }
$$

where $x_{\mathrm{cm}}$ is the position of the center of mass, $x_{i}$ is the position of candidate solution $i$ in $n$ dimensional search space, $f_{i}$ is the penalized fitness function value of candidate solution I, and $\mathrm{np}$ is the size of the initial population.

(3) Compute the position of the candidate solutions in the next iteration using the following expression considering that they are normally distributed around the center of mass $x_{\mathrm{cm}}$ :

$$
x_{i}^{\text {next }}=x_{\mathrm{cm}}+\frac{r \alpha\left(x_{\max }-x_{\min }\right)}{s},
$$

where $x_{i}^{\text {next }}$ is the position of the new candidate solution $i, r$ is the random number from a standard normal distribution, $\alpha$ is the parameter that limits the size of the search space, $x_{\max }$ and $x_{\min }$ are the upper and lower bounds on the design variables, and $s$ is the number of big bang iterations. In the case where steel sections are to be selected from the available steel sections list then it becomes necessary to work with integer numbers. In this case $x_{i}^{\text {next }}$ of (67) is rounded to the nearest integer number as $I_{i}^{\text {next }}=$ $\operatorname{ROUND}\left(x_{i}^{\text {next }}\right)$ where $I_{i}^{\text {next }}$ represents index number the steel profile from the tabular discrete list.

(4) Repeat steps 2 and 3 until termination criteria are satisfied.
Camp [223] developed optimum design algorithm for space trusses based on big bang-big crunch optimizer. The number of benchmark design examples having design variables continuous as well discrete is taken from the literature and designed with the developed algorithm. The results are compared with those algorithms of quadratic programming, general geometric programming, genetic algorithm, particle swarm optimizer, and ant colony optimization. It is reported that big bang-big crunch optimizer has relatively small number of parameters to define which provides the algorithm with better performance over the other techniques considered in the study. It is also concluded that the algorithm showed significant improvements in the consistency and computational efficiency when compared to genetic algorithm, particle swarm optimizer, and ant colony technique.

Kaveh and Talatahari [224] also presented big bangbig crunch-based optimum design algorithm for size optimization of space trusses. In this study large size space trusses are designed by the algorithm developed as well as those stochastic search techniques of genetic algorithm, ant colony optimization, particle swarm optimizer, and standard harmony search method. It is stated that big bang-big crunch algorithm performs well in the optimum design of largesize space trusses contrary to other metaheuristic techniques which presents convergence difficulty or get trapped at a local optimum.

Kaveh and Talatahari [225] developed optimum topology design algorithm based on hybrid big bang-big crunch optimization method for schwedler and ribbed domes. The algorithm determines the optimum configuration as well as optimum member sizes of these domes. It is reported that big bang-big crunch optimization method efficiently determined the optimum solution of large dome structures.

Kaveh and Abbasgholiha [226] presented the optimum design algorithm for steel frames based on big bang-big crunch optimizer. The design problem is formulated according to BS5950, ASD-AISCF, and LRFD-AISC design codes, and the optimum results obtained by each code are compared. It is stated that LRFD design codes yield to the lightest steel frame as expected among other codes.

5.7. Hybrid and Other Stochastic Search Techniques. Stochastic search techniques have two drawbacks although they are capable of determining the optimum solution of discrete structural optimization problems. First one is that there is no guarantee that the solution found at the end of predetermined number of iterations is the global optimum. There is no mathematical proof available in these techniques due to the fact that they use heuristics not mathematically derived expression. The optimum solution obtained may very well be a local optimum or near optimum solution. Second drawback is that they need large amount of structural analysis to reach the near optimum solution. Some work is carried out to improve the performance of the metaheuristic optimization techniques by hybridizing them. Some of these algorithms are reviewed below.

Kaveh and Talatahari $[227,228]$ developed hybrid particle swarm and ant colony optimization algorithm for the discrete 
optimum design of steel frames. The algorithm uses particle swarm optimizer for global search and ant colony optimization for local search. It is reported that the hybrid algorithm is quite effective in finding the optimum solutions.

Kaveh and Talatahari $[229,230]$ have also combined the search strategies of the harmony search method, particle swarm optimizer, and ant colony optimization to obtain efficient metaheuristic technique called HPSACO for the optimum design of steel frames. In this technique particle swarm optimization with passive congregation is used for global search, and the ant colony optimization is employed for local search. The harmony search based mechanism is utilized to handle the variable constraints. It is demonstrated in the design examples considered that proposed hybrid technique finds lighter optimum designs than each standard particle swarm optimizer and ant colony optimization as well as harmony search method. Further improvements are suggested for the algorithm in [231].

Kaveh and Rad [232] presented another hybrid genetic algorithm and particle swarm optimization technique for the optimum design of steel frames. They have introduced the maturing phenomenon which is mimicked by particle swarm optimizer where individuals enhance themselves based on social interactions and their private cognition. Crossover is applied to this society. Hence evolution of individuals is no longer restricted to the same generation. The results obtained from the design examples have shown that the hybrid algorithm shows superiority in optimum design of large-size steel frames.

Kaveh and Talatahari [233] presented a structural optimization method based on sociopolitically motivated strategy called imperialist competitive algorithm. Imperialist competitive algorithm initiates the search by considering multiagents where each agent is considered to be a country which is either a colony or an imperialist. Countries form colonies in the search space, and they move towards their related empires. During this movement, weak empires collapses and strong ones get stronger. Such movements direct the algorithm to optimum point. Presented algorithm is used in the optimum design of skeletal steel frames.

Kaveh and Talatahari [234] also introduced a novel heuristic search technique based on some principles of physics and mechanics called charged system search. The method is a multiagent approach where each agent is a charged particle. These particles affect each other based on their fitness values and distances among them. The quantity of the resultant force is determined by using electrostatic laws, and the quality of movement is determined using Newtonian mechanics laws. It is stated that charged system search algorithm is compared with other metaheuristic algorithm on benchmark examples, and it is found that it outperformed the others. The algorithm is applied to optimum design of skeletal structures in $[235,236]$, to grillage systems in [236], to truss structures in [237], and to geodesic dome in [238]. It is stated in all these works that charged system search algorithm shows better performance than other metaheuristic techniques considered. In [239] an improvement is suggested for the algorithm to enhance its performance even further.
The algorithm is applied to optimum design of steel frames in [240].

Kaveh and Bakhspoori [241] used cuckoo search method to develop optimum design algorithm for steel frames. The design problem is formulated according to Load and Resistance Factor Design code of American Institute of Steel Construction [72]. The optimum designs obtained by cuckoo search algorithm are compared with those attained by other algorithms on benchmark frames. Cuckoo search algorithm is originated by Yang and Deb [242] which simulates reproduction strategy of cuckoo birds. Some species of cuckoo birds lay their eggs in the nests of other birds so that when the eggs are hatched their chicks are fed by the other birds. Sometimes they even remove existing eggs of host nest in order to give more probability of hatching of their own eggs. Some species of cuckoo birds are even specialized to mimic the pattern and color of the eggs of host birds so that host bird could not recognize their eggs which gives more possibility of hatching. In spite of all these efforts to conceal their eggs from the attention of host birds, there is a possibility that host bird may discover that the eggs are not its own. In such cases the host bird either throws these alien eggs away or simply abandons its nest and builds a new nest somewhere else. The engineering design optimization of cuckoo search algorithm is carried out in [243].

5.8. Evaluation of Stochastic Search Techniques. It is apparent that there are a lot of metaheuristic algorithms that can be used in the optimum design of steel frames. The question of which one of these algorithms outperforms the others requires an answer. It should be pointed out that the performance of metaheuristic algorithms is dependent upon the selection of the initial values for their parameters which is quite problem dependent. The following works try to provide an answer to the previously mentioned problem.

Hasançebi et al. [244, 245] evaluated the performance of the stochastic search algorithm used in structural optimization on the large-scale pin jointed and rigidly jointed steel frames. Among these techniques genetic algorithms, simulated annealing, evolution strategies, particle swarm optimizer, tabu search, ant colony optimization, and harmony search method are utilized to develop seven optimum design algorithms for real-size pin and rigidly connected large-scale steel frames. The design problems are formulated according to ASD-AISC (Allowable Stress Design Code of American Institute of Steel Institution). The results reveal that simulated annealing and evolution strategies are the most powerful techniques, and standard harmony search and simple genetic algorithm methods can be characterized by slow convergence rates and unreliable search performance in large-scale problems.

Kaveh and Talatahari [246] used particle swarm optimizer, ant colony optimization, harmony search method, big bang-big crunch, hybrid particle swarm ant colony optimization, and charged system search techniques in the optimum design of single-layer Schwedler and lamella domes. The design problem is formulated according to LRFD-AISC specifications. It is stated that among these algorithm hybrid 
particle swarm, ant colony optimization, and charged system search algorithms have illustrated better performance compared to other heuristic algorithms.

\section{Conclusions}

The review carried out reveals the fact that the mathematical modeling of the optimum design problem of steel frames can be broadly formulated in different ways. In the first way the cross-sectional properties of frame members treated only the optimization variables. In this case joint displacements are not part of optimization model, and their values are required to be obtained through structural analysis in every design cycle. This type of formulation is called coupled analysis and design (CAND). Naturally in this the type of formulation the total number of analysis is large which is computationally inefficient. In the second type of formulation the joint displacements are also treated as optimization variables in addition to cross-sectional properties. This makes it necessary to include the stiffness equations as constraints in the mathematical model as equality constraints. Such formulation is called simultaneous analysis and design (SAND) which eliminates the necessity of carrying out structural analysis in every design cycle. Hence the total number of structural analysis required to reach the optimum solution becomes quite less compared to the first type of modeling. However, in the second type the total number of optimization variables is quite large, and it becomes necessary to utilize powerful optimization techniques that work efficiently in solving large-size optimization problems.

The design code that is to be considered in the modeling of the optimum design problem also affects the complexity of the optimization problem obtained. Formulating the optimum design of steel frames without referencing any design code brings out relatively simple optimization problem if linear elastic structural behavior is assumed. Furthermore, if continuous design variables assumption is also made, then mathematical programming or optimality criteria algorithms efficiently finds the optimum solution of the optimization problem in both ways of modeling. Optimality criteria algorithms also provide optimum solutions without any difficulty even if nonlinear elastic behavior is considered in the optimum design of steel frames. Among the mathematical programming techniques, it seems that sequential quadratic programming method is the most powerful, and it is reported in several works that it can attain the optimum solution without any difficulty in large-size steel frame design optimization. If mathematical modeling of the frame design optimization problem is to be formulated such that the allowable stress design code specifications such as the displacement and stress limitations are required to be satisfied in the optimum design the optimality criteria approaches provide efficient algorithms for that purpose. However, it should be pointed out that in the optimum solution the design variables will have values attained from a continuous variables assumption. On the other hand practicing structural designer needs cross-sectional properties selected from the available steel sections list. This necessitates first finding the continuous optimum solution and then round these to the nearest available values. Such move may yield loss of what is gained through optimization. Altering the mathematical programming or optimality criteria technique to work with discrete variables makes the algorithms complicated, and they present difficulties in obtaining the optimum solution of real-size steel frames.

Emergence of the stochastic search techniques provides steel frame designers with new capabilities. These new techniques do not need the gradient calculations of objective function and constraints. They do not use mathematical derivation in order to find a way to reach the optimum. Instead they rely on heuristics. These new optimization techniques use nature as a source of inspiration to develop numerical optimization procedures that are capable of solving complex engineering design problems. They are particularly efficient in obtaining the optimum solution where the design variables are to be selected from a tabulated list. As summarized in this paper there are several stochastic search techniques that are successfully used in the optimum design steel frames where the steel sections for the frame members are to be selected from the available steel sections list, while the limit state design code specifications such as serviceability and strength are to be satisfied. These techniques do provide optimum solution that can be directly used by the practicing designers in their projects. However, they also have some drawbacks. The first one is that because they do not use mathematical derivations; it is not possible to prove whether the optimum solution they attain is the global optimum or it is near optimum. The second is that they work with random numbers, and they have a number of parameters which need to be given values by the users prior to their application. Selection of these values affects the performance of algorithms. This requires a sensitivity works with different values of these parameters in order to find the appropriate values for the problem under consideration. Although some techniques are available such as adaptive genetic algorithm and adaptive harmony search method where the values of these parameters are adjusted automatically by the algorithm itself, this is not the case for other techniques. The third drawback is that they need a large number of structural analysis which becomes computationally very expensive for largesize steel frames. Among the existing techniques some of them excel and outperform others. It is apparent that further research is required to reduce the total number of structural analysis required by stochastic search algorithm which is computationally expensive to a reasonable amount. However, the search for finding better stochastic search techniques is continuing. It is difficult at this moment to conclude which one of these techniques will become the standard one that will be used in the design tools of the finite element packages that are used in everyday practice. However, it is not difficult to conclude that metaheuristic techniques are going to be standard design optimization tools in the near future.

\section{Acknowledgments}

This work was supported by the Gachon University Research Fund of 2012 (GCU-2012-R259). However, there is no conflict 
of interests which exists when professional judgment concerning the validity of research is influenced by a secondary interest, such as financial gain.

\section{References}

[1] L. A. Schmit, "Structural Design by Systematic Synthesis," in Proceedings of the ASCE 2nd Conference on Electronic Computation, pp. 105-132, 1960.

[2] K. I. Majid, Optimum Design of Structures, Butterworths, London, UK, 1974.

[3] M. P. Saka, Optimum design of structures [Ph.D. thesis], University of Aston, Birmingham, UK, 1975.

[4] L. A. A. Schmit and H. Miura, "Approximating concepts for efficient structural synthesis," Tech. Rep. NASA CR-2552, 1976.

[5] M. P. Saka, "Optimum design of rigidly jointed frames," Computers and Structures, vol. 11, no. 5, pp. 411-419, 1980.

[6] E. Atrek, R. H. Gallagher, K. M. Ragsdell, and O. C. Zienkewics, Eds., New Directions in Optimum Structural Design, John Wiley \& Sons, Chichester, UK, 1984.

[7] R. T. Haftka, "Simultaneous analysis and design," AIAA Journal, vol. 23, no. 7, pp. 1099-1103, 1985.

[8] J. S. Arora, Introduction to Optimum Design, McGraw-Hill, 1989.

[9] R. T. Haftka and Z. Gurdal, Elements of Structural Optimization, Kluwer Academic Publishers, The Netherlands, 1992.

[10] U. Kirsch, Structural Optimization, Springer, 1993.

[11] J. S. Arora, "Guide to structural optimization," ASCE Manuals and Reports on Engineering Practice number 90, ASCE, New York, NY, USA, 1997.

[12] A. D. Belegundi and T. R. Chandrupath, Optimization Concepts and Application in Engineering, Prentice-Hall, 1999.

[13] American Institutes of Steel Construction, Manual of Steel Construction, Allowable Stress Design, American Institutes of Steel Construction, Chicago, Ill, USA, 9th edition, 1989.

[14] M. P. Saka, "Optimum design of steel frames with stability constraints," Computers and Structures, vol. 41, no. 6, pp. 13651377, 1991.

[15] M. P. Saka, “Optimum design of skeletal structures: a review," in Progress in Civil and Structural Engineering Computing, H. V. B. Topping, Ed., chapter 10, pp. 237-284, Saxe-Coburg, 2003.

[16] M. P. Saka, "Optimum design of steel frames using stochastic search techniques based on natural phenoma: a review," in Civil Engineering Computations: Tools and Techniques, B. H. V. Topping, Ed., chapter 6, pp. 105-147, Saxe-Coburg, 2007.

[17] J. S. Arora and Q. Wang, "Review of formulations for structural and mechanical system optimization," Structural and Multidisciplinary Optimization, vol. 30, no. 4, pp. 251-272, 2005.

[18] J. S. Arora, "Methods for discrete variable structural optimization," in Recent Advances in Optimum Structural Design, S. A. Burns, Ed., pp. 1-40, ASCE, USA, 2002.

[19] R. E. Griffith and R. A. Stewart, "A non-linear programming technique for the optimization of continuous processing systems," Management Science, vol. 7, no. 4, pp. 379-392, 1961.

[20] M. P. Saka, "The use of the approximate programming in the optimum structural design," in Proceedings of International Conference on Mathematics, Black Sea Technical University, Trabzon, Turkey, September, 1982.

[21] K. F. Reinschmidt, C. A. Cornell, and J. F. Brotchie, "Iterative design and structural optimization," Journal of Structural Division, vol. 92, pp. 319-340, 1966.
[22] G. Pope, "Application of linear programming technique in the design of optimum structures," in Proceedings of the AGAR Symposium Structural Optimization, Istanbul, Turkey, October, 1969.

[23] D. Johnson and D. M. Brotton, "Optimum elastic design of redundant trusses," Journal of the Structural Division, vol. 95, no. 12, pp. 2589-2610, 1969.

[24] J. S. Arora and E. J. Haug, "Efficient optimal design of structures by generalized steepest descent programming," International Journal for Numerical Methods in Engineering, vol. 10, no. 4, pp. 747-766, 1976.

[25] P. E. Gill, W. Murray, and M. H. Wright, Practical Optimization, Academic Press, 1981.

[26] J. Nocedal and J. S. Wright, Numerical optimization, Springer, New York, NY, USA, 1999.

[27] S. P. Han, "A globally convergent method for nonlinear programming," Journal of Optimization Theory and Applications, vol. 22, no. 3, pp. 297-309, 1977.

[28] M.-W. Huang and J. S. Arora, "A self-scaling implicit SQP method for large scale structural optimization," International Journal for Numerical Methods in Engineering, vol. 39, no. 11, pp. 1933-1953, 1996.

[29] Q. Wang and J. S. Arora, "Alternative formulations for structural optimization: an evaluation using frames," Journal of Structural Engineering, vol. 132, no. 12, Article ID 008612QST, pp. 18801889, 2006.

[30] Q. Wang and J. S. Arora, "Optimization of large-scale truss structures using sparse SAND formulations," International Journal for Numerical Methods in Engineering, vol. 69, no. 2, pp. 390407, 2007.

[31] C. W. Carroll, "The crated response surface technique for optimizing nonlinear restrained systems," Operations Research, vol. 9, no. 2, pp. 169-184, 1961.

[32] A. V. Fiacco and G. P. McCormack, Nonlinear Programming Sequential Unconstrained Minimization Techniques, John Wiley \& Sons, New York, NY, USA, 1968.

[33] D. Kavlie and J. Moe, "Automated design of framed structures," Journal of Structural Division, vol. 97, no. 1, 33 pages, 62.

[34] D. Kavlie and J. Moe, "Application of non-linear programming to optimum grillage design with non-convex sets of variables," International Journal for Numerical Methods in Engineering, vol. 1, no. 4, pp. 351-378, 1969.

[35] K. M. Griswold and J. Moe, "A method for non-linear mixed integer programming and its application to design problems," Journal of Engineering for Industry, vol. 94, no. 2, 12 pages, 1972.

[36] B. M. E. de Silva and G. N. C. Grant, "Comparison of some penalty function based optimization procedures for synthesis of a planar truss," International Journal for Numerical Methods in Engineering, vol. 7, no. 2, pp. 155-173, 1973.

[37] J. B. Rosen, "The gradient-projection method for nonlinear programming-Part II," Journal of the Society for Industrial and Applied Mathematics, vol. 9, pp. 514-532, 1961.

[38] E. J. Haug and J. S. Arora, Applied Optimal Design, John Wiley, New York, NY, USA, 1979.

[39] G. Zoutindijk, Methods of Feasible Directions, Elsevier Science, Amsterdam, The Netherlands, 1960.

[40] G.N. Vanderplaats, "CONMIN: a fortran program for constrained function minimization," Tech. Rep. NASA TNX-62282, 1973.

[41] G. N. Vanderplaats, Numerical Optimization Techniques for Engineering Design, McGraw-Hill, New York, NY, USA, 1984. 
[42] C. Zener, Engineering Design by Geometric Programming, JohnWiley, London, UK, 1971.

[43] A. J. Morris, "Structural optimization by geometric programming," in Foundation in Structural Optimization: A Unified Approach, A. J. Morris, Ed., pp. 573-610, John Wiley \& Sons, Chichester, UK, 1982.

[44] R.E. Bellman, Dynamic Programming, Princeton University Press, Princeton, NJ, USA, 1957.

[45] A.C. Palmer, "Optimum structural design by dynamic programming," Journal of the Structural Division, vol. 94, pp. 18871906, 1968.

[46] D. J. Sheppard and A. C. Palmer, "Optimal design of transmission towers by dynamic programming," Computers and Structures, vol. 2, no. 4, pp. 455-468, 1972.

[47] D. M. Himmelblau, Applied Nonlinear Programming, McGrawHill, New York, NY, USA, 1972.

[48] E. D. Eason and R. G. Fenton, "Comparison of numerical optimization methods for engineering design," Journal of Engineering for Industry, Series B, vol. 96, no. 1, pp. 196-200, 1974.

[49] W. Prager, "Optimality criteria in structural design," Proceedings of National Academy for Science, vol. 61, no. 3, pp. 794-796, 1968.

[50] V. B. Venkayya, N. S. Khot, and V. S. Reddy, "Energy distribution in optimum structural design," Tech. Rep. AFFDL-TM-68-156, Flight Dynamics laboratory, Wright Patterson, AFB Ohio, USA, 1968.

[51] V. B. Venkayya, N. S. Khot, and L. Berke, "Application of optimality criteria approaches to automated design of large practical structures," in Proceedings of the 2nd Symposium on Structural Optimization, AGARD-CP-123, pp. 3-1-3-19, 1973.

[52] V. B. Venkayya, "Optimality criteria: a basis for multidisciplinary design optimization," Computational Mechanics, vol. 5, no. 1, pp. 1-21, 1989.

[53] L. Berke, "An efficient approach to the minimum weight design of deflection limited structures," Tech. Rep. AFFDL-TM-70-4FDTR, 1970.

[54] L. Berke and N.S. Khot, "Structural optimization using optimality criteria," in Computer Aided Structural Design, C. A. M. Soares, Ed., Springer, 1987.

[55] N. S. Khot, L. Berke, and V. B. Venkayya, "comparison of optimality criteria algorithms for minimum weight design of structures," AIAA Journal, vol. 17, no. 2, pp. 182-190, 1979.

[56] N. S. Khot, "algorithms based on optimality criteria to design minimum weight structures," Engineering Optimization, vol. 5, no. 2, pp. 73-90, 1981.

[57] N. S. Khot, "Optimal design of a structure for system stability for a specified eigenvalue distribution," in New Directions in Optimum Structural Design, E. Atrek, R. H. Gallagher, K. M. Ragsdell, and O. C. Zienkiewicz, Eds., pp. 75-87, John Wiley \& Sons, New York, NY, USA, 1984.

[58] K.M. Ragsdell, "Utility of nonlinear programming methods for engineering design," in New Direction in Optimality Criteria in Structural Design, E. Atrek et al., Ed., Chapter 18, John Wiley \& Sons, 1984.

[59] C. Feury and M. Geradin, "Optimality criteria and mathematical programming in structural weight optimization," Journal of Computers and Structures, vol. 8, no. 1, pp. 7-17, 1978.

[60] C. Fleury, "An efficient optimality criteria approach to the minimum weight design of elastic structures," Journal of Computers and Structures, vol. 11, no. 3, pp. 163-173, 1980.

[61] M. P. Saka, "Optimum design of space trusses with buckling constraints," in Proceedings of 3rd International Conference on Space Structures, University of Surrey, Guildford, U.K., September 1984.

[62] E. I. Tabak and P. M. Wright, "Optimality criteria method for building frames," Journal of Structural Division, vol. 107, no. 7, pp. 1327-1342, 1981.

[63] F. Y. Cheng and K. Z. Truman, "Optimization algorithm of 3-D building systems for static and seismic loading," in Modeling and Simulation in Engineering, W. F. Ames, Ed., pp. 315-326, NorthHolland, Amsterdam, The Netherlands, 1983.

[64] M. R. Khan, "Optimality criterion techniques applied to frames having general cross-sectional relationships," AIAA Journal, vol. 22, no. 5, pp. 669-676, 1984.

[65] E. A. Sadek, "Optimization of structures having general crosssectional relationships using an optimality criterion method," Computers and Structures, vol. 43, no. 5, pp. 959-969, 1992.

[66] M. P. Saka, "Optimum design of pin-jointed steel structures with practical applications," Journal of Structural Engineering, vol. 116, no. 10, pp. 2599-2620, 1990.

[67] C. G. Salmon, J. E. Johnson, and F. A. Malhas, Steel Structures: Design and Behavior, Prentice Hall, New York, NY, USA, 5th edition, 2009.

[68] D. E. Grieson and G. E. Cameron, SODA-Structural Optimization Design and Analysis, Release 3. 1, User manual, Waterloo Engineering Software, Waterloo, Canada, 1990.

[69] M. P. Saka, "Optimum design of multistorey structures with shear walls," Computers and Structures, vol. 44, no. 4, pp. 925936, 1992.

[70] M. P. Saka, "Optimum geometry design of roof trusses by optimality criteria method," Computers and Structures, vol. 38, no. 1, pp. 83-92, 1991.

[71] M. P. Saka, "Optimum design of steel frames with tapered members," Computers and Structures, vol. 63, no. 4, pp. 797-811, 1997.

[72] AISC Manual Committee, "Load and resistance factor design," in Manual of Steel Construction, AISC, USA, 1999.

[73] S. S. Al-Mosawi and M. P. Saka, "Optimum design of single core shear walls," Computers and Structures, vol. 71, no. 2, pp. 143$162,1999$.

[74] S. Al-Mosawi and M. P. Saka, "Optimum shape design of coldformed thin-walled steel sections," Advances in Engineering Software, vol. 31, no. 11, pp. 851-862, 2000.

[75] V. Z. Vlasov, Thin-Walled Elastic Beams, National Science Foundation, Wash, USA, 1961.

[76] C.-M. Chan and G. E. Grierson, "An efficient resizing technique for the design of tall steel buildings subject to multiple drift constraints," in The Structural Design of Tall Buildings, vol. 2, no. 1, pp. 17-32, John Wiley \& Sons, West Sussex, UK, 1993.

[77] C.-M. Chan, "How to optimize tall steel building frameworks," in Guide To Structural Optimization, ASCE Manuals and Reports on Engineering Practice, J. S. Arora, Ed., no. 90, Chapter 9, pp. 165-196, ASCE, New York, NY, USA, 1997.

[78] R. Soegiarso and H. Adeli, "Optimum load and resistance factor design of steel space-frame structures," Journal of Structural Engineering, vol. 123, no. 2, pp. 184-192, 1997.

[79] N. S. Khot, "Nonlinear analysis of optimized structure with constraints on system stability," AIAA Journal, vol. 21, no. 8, pp. 1181-1186, 1983.

[80] N. S. Khot and M. P. Kamat, "Minimum weight design of truss structures with geometric nonlinear behavior," AIAA Journal, vol. 23, no. 1, pp. 139-144, 1985. 
[81] M. P. Saka, "Optimum design of nonlinear space trusses," Computers and Structures, vol. 30, no. 3, pp. 545-551, 1988.

[82] M. P. Saka and M. Ulker, "Optimum design of geometrically nonlinear space trusses," Computers and Structures, vol. 42, no. 3, pp. 289-299, 1992.

[83] M. P. Saka and M. S. Hayalioglu, "Optimum design of geometrically nonlinear elastic-plastic steel frames," Computers and Structures, vol. 38, no. 3, pp. 329-344, 1991.

[84] M. S. Hayalioglu and M. P. Saka, "Optimum design of geometrically nonlinear elastic-plastic steel frames with tapered members," Computers and Structures, vol. 44, no. 4, pp. 915-924, 1992.

[85] M. P. Saka and E. S. Kameshki, "Optimum design of unbraced rigid frames," Computers and Structures, vol. 69, no. 4, pp. 433442, 1998.

[86] M. P. Saka and E. S. Kameshki, "Optimum design of nonlinear elastic framed domes," Advances in Engineering Software, vol. 29, no. 7-9, pp. 519-528, 1998.

[87] J. H. Holland, Adaptation in Natural and Artificial Systems, The University of Michigan press, Ann Arbor, Minn, USA, 1975.

[88] S. Kirkpatrick, C. D. Gerlatt, and M. P. Vecchi, "Optimization by simulated annealing," Science, vol. 220, pp. 671-680, 1983.

[89] D. E. Goldberg and M. P. Santani, "Engineering optimization via generic algorithm," in Proceedings of 9th Conference on Electronic Computation, pp. 471-482, ASCE, New York, NY, USA, 1986.

[90] D. E. Goldberg, Genetic Algorithms in Search, Optimization and Machine Learning, Addison Wesley, 1989.

[91] R. Paton, Computing With Biological Metaphors, Chapman \& Hall, New York, NY, USA, 1994.

[92] R. Horst and P. M. Pardolos, Eds., Handbook of Global Optimization, Kluwer Academic Publishers, New York, NY, USA, 1995.

[93] R. Horst and H. Tuy, Global Optimization, Deterministic Approaches, Springer, 1995.

[94] C. Adami, An Introduction to Artificial Life, Springer-Telos, 1998.

[95] C. Matheck, Design in Nature: Learning from Trees, Springer, Berlin, Germany, 1998.

[96] M. Mitchell, An Introduction to Genetic Algorithms, The MIT Press, Boston, Mass, USA, 1998.

[97] G. W. Flake, The Computational Beauty of Nature, MIT Press, Boston, Mass, USA, 2000.

[98] L. Chambers, The Practical Handbook of Genetic Algorithms, Applications, Chapman \& Hall, 2nd edition, 2001.

[99] J. Kennedy, R. Eberhart, and Y. Shi, Swarm Intelligence, Morgan Kaufmann, Boston, Mass, USA, 2001.

[100] G. A. Kochenberger and F. Glover, Handbook of MetaHeuristics, Kluwer Academic Publishers, New York, NY, USA, 2003.

[101] L. N. De Castro and F. J. Von Zuben, Recent Developments in Biologically Inspired Computing, Idea Group Publishing, USA, 2005.

[102] J. Dreo, A. Petrowski, P. Siarry, and E. Taillard, Meta-Heuristics for Hard Optimization, Springer, Berlin, Germany, 2006.

[103] T. F. Gonzales, Handbook of Approximation Algorithms and Metaheuristics, Chapman \& Halls/CRC, 2007.

[104] X.-S. Yang, Nature-Inspired Metaheuristic Algorithms, Luniver Press, 2008.

[105] X. -S. Yang, Engineering Optimization: An Introduction With Metaheuristic Applications, John Wiley, New York, NY, USA, 2010.
[106] S. Luke, "Essentials of Metaheuristics," 2010, http://cs.gmu.edu/ $\sim$ sean/book/metaheuristics/.

[107] P. Lu, S. Chen, and Y. Zheng, "Artificial intelligence in civil engineering," Mathematical Problems in Engineering, vol. 2012, Article ID 145974, 22 pages, 2012.

[108] R. Kicinger, T. Arciszewski, and K. De Jong, "Evolutionary computation and structural design: a survey of the state-of-theart," Computers and Structures, vol. 83, no. 23-24, pp. 1943-1978, 2005.

[109] I. Rechenberg, "Cybernetic solution path of an experimental problem," in ,Royal Aircraft Establishment, no. 1122, Library Translation, Farnborough, UK, 1965.

[110] I. Rechenberg, Evolutionsstrategie: optimierung technischer systeme nach prinzipen der biologischen evolution, FrommannHolzboog, Stuttgart, Germany, 1973.

[111] H. -P. Schwefel, "Kybernetische evolution als strategie der experimentellen forschung in der strömungstechnik," in Diplomarbeit, Technishe Universitat, Berlin, Germany, 1965.

[112] D. E. Goldberg and M. P. Santani, "Engineering optimization via generic algorithm," in Proceedings of 9th Conference on Electronic Computation, pp. 471-482, ASCE, New York, NY, USA, 1986.

[113] M. Gen and R. Chang, Genetic Algorithm and Engineering Design, John Wiley \& Sons, New York, NY, USA, 2000.

[114] D. E. Goldberg and M. P. Santani, "Engineering optimization via generic algorithm," in Proceedings of 9th Conference on Electronic Computation, pp. 471-482, ASCE, New York, NY, USA, 1986.

[115] S. Rajev and C. S. Krishnamoorthy, "Discrete optimization of structures using genetic algorithms," Journal of Structural Engineering, vol. 118, no. 5, pp. 1233-1250, 1992.

[116] F. Herrera, M. Lozano, and J. L. Verdegay, “Tackling realcoded genetic algorithms: operators and tools for behavioural analysis," Artificial Intelligence Review, vol. 12, no. 4, pp. 265319, 1998.

[117] J. P. B. Leite and B. H. V. Topping, "Improved genetic operators for structural engineering optimization," Advances in Engineering Software, vol. 29, no. 7-9, pp. 529-562, 1998.

[118] W. M. Jenkins, “Towards structural optimization via the genetic algorithm," Computers and Structures, vol. 40, no. 5, pp. 13211327, 1991.

[119] W. M. Jenkins, "Structural optimization via the genetic algorithm," The Structural Engineer, vol. 69, no. 24, pp. 418-422, 1991.

[120] P. Hajela, "Stochastic search in structural optimization: genetic algorithms and simulated annealing," in Structural Optimization: Status and Promise, chapter 22, pp. 611-635, American Institute of Aeronautics and Astronautics, 1992.

[121] H. Adeli and N. T. Cheng, "Augmented lagrange genetic algorithm for structural optimization," Journal of Structural Engineering, vol. 7, no. 3, pp. 104-118, 1994.

[122] H. Adeli and N. T. Cheng, "Concurrent genetic algorithms for optimization of large structures," Journal of Aerospace Engineering, vol. 7, no. 3, pp. 276-296, 1994.

[123] S. D. Rajan, "Sizing, shape, and topology design optimization of trusses using genetic algorithm," Journal of Structural Engineering, vol. 121, no. 10, pp. 1480-1487, 1995.

[124] C. V. Camp, S. Pezeshk, and G. Cao, "Design of framed structures using a genetic algorithm," in Advances in Structural Optimization, D. M. Frangopol and F. Y. Cheng, Eds., pp. 19-30, ASCE, NY, USA, 1997. 
[125] C. Camp, S. Pezeshk, and G. Cao, "Optimized design of twodimensional structures using a genetic algorithm," Journal of Structural Engineering, vol. 124, no. 5, pp. 551-559, 1998.

[126] M. P. Saka and E. Kameshki, "Optimum design of multi-storey sway steel frames to BS5950 using a genetic algorithm," in Advances in Engineering Computational Technology, B. H. V. Topping, Ed., pp. 135-141, Civil-Comp Press, Edinburgh, UK, 1998.

[127] M. P. Saka, "Optimum design of grillage systems using genetic algorithms," Computer-Aided Civil and Infrastructure Engineering, vol. 13, no. 4, pp. 297-302, 1998.

[128] S. H. Weldali and M. P. Saka, "Optimum geometry and spacing design of roof trusses based on BS5990 using genetic algorithm," Design Optimization, vol. 1, no. 2, pp. 198-219, 2000.

[129] M. P. Saka, A. Daloglu, and F. Malhas, "Optimum spacing design of grillage systems using a genetic algorithm," Advances in Engineering Software, vol. 31, no. 11, pp. 863-873, 2000.

[130] S. Pezeshk, C. V. Camp, and D. Chen, "Design of nonlinear framed structures using genetic optimization," Journal of Structural Engineering, vol. 126, no. 3, pp. 387-388, 2000.

[131] O. Hasançebi and F. Erbatur, "Evaluation of crossover techniques in genetic algorithm based optimum structural design," Computers and Structures, vol. 78, no. 1, pp. 435-448, 2000.

[132] F. Erbatur, O. Hasançebi, İ. Tütüncü, and H. Kılıç, "Optimal design of planar and space structures with genetic algorithms," Computers and Structures, vol. 75, pp. 209-224, 2000.

[133] E. S. Kameshki and M. P. Saka, "Genetic algorithm based optimum bracing design of non-swaying tall plane frames," Journal of Constructional Steel Research, vol. 57, no. 10, pp. 10811097, 2001.

[134] E. S. Kameshki and M. P. Saka, "Optimum design of nonlinear steel frames with semi-rigid connections using a genetic algorithm," Computers and Structures, vol. 79, no. 17, pp. 1593-1604, 2001.

[135] J. Andre, P. Siarry, and T. Dognon, "An improvement of the standard genetic algorithm fighting premature convergence in continuous optimization," Advances in Engineering Software, vol. 32, no. 1, pp. 49-60, 2001.

[136] V. V. Toropov and S. Y. Mahfouz, "Design optimization of structural steelwork using a genetic algorithm, FEM and a system of design rules," Engineering Computations, vol. 18, no. 3-4, pp. 437-459, 2001.

[137] M. S. Hayalioğlu, "Optimum load and resistance factor design of steel space frames using genetic algorithm," Structural and Multidisciplinary Optimization, vol. 21, no. 4, pp. 292-299, 2001.

[138] W. M. Jenkins, "A decimal-coded evolutionary algorithm for constrained optimization," Computers and Structures, vol. 80, no. 5-6, pp. 471-480, 2002.

[139] E. S. Kameshki and M. P. Saka, "Genetic algorithm based optimum design of nonlinear planar steel frames with various semirigid connections," Journal of Constructional Steel Research, vol. 59, no. 1, pp. 109-134, 2003.

[140] Y. M. Yun and B. H. Kim, "Optimum design of plane steel frame structures using second-order inelastic analysis and a genetic algorithm," Journal of Structural Engineering, vol. 131, no. 12, pp. 1820-1831, 2005.

[141] A. Kaveh and M. Shahrouzi, "Simultaneous topology and size optimization of structures by genetic algorithm using minimal length chromosome," Engineering Computations, vol. 23, no. 6, pp. $644-674,2006$.
[142] R. J. Balling, R. R. Briggs, and K. Gillman, "Multiple optimum size/shape/topology designs for skeletal structures using a genetic algorithm," Journal of Structural Engineering, vol. 132, no. 7, Article ID 015607QST, pp. 1158-1165, 2006.

[143] V. Toğan and A. T. Daloğlu, "Optimization of 3D trusses with adaptive approach in genetic algorithms," Engineering Structures, vol. 28, pp. 1019-1027, 2006.

[144] E. S. Kameshki and M. P. Saka, "Optimum geometry design of nonlinear braced domes using genetic algorithm," Computers and Structures, vol. 85, no. 1-2, pp. 71-79, 2007.

[145] S. O. Degertekin, "A comparison of simulated annealing and genetic algorithm for optimum design of nonlinear steel space frames," Structural and Multidisciplinary Optimization, vol. 34, no. 4, pp. 347-359, 2007.

[146] S. O. Degertekin, M. P. Saka, and M. S. Hayalioglu, "Optimal load and resistance factor design of geometrically nonlinear steel space frames via tabu search and genetic algorithm," Engineering Structures, vol. 30, no. 1, pp. 197-205, 2008.

[147] H. K. Issa and F. A. Mohammad, "Effect of mutation schemes on convergence to optimum design of steel frames," Journal of Constructional Steel Research, vol. 66, no. 7, pp. 954-961, 2010.

[148] D. Safari, M. R. Maheri, and A. Maheri, "Optimum design of steel frames using a multiple-deme GA with improved reproduction operators," Journal of Constructional Steel Research, vol. 67, no. 8, pp. 1232-1243, 2011.

[149] N. D. Lagaros, M. Papadrakakis, and G. Kokossalakis, "Structural optimization using evolutionary algorithms," Computers and Structures, vol. 80, no. 7-8, pp. 571-589, 2002.

[150] J. Cai and G. Thierauf, "Discrete structural optimization using evolution strategies," in Neural Networks and Combinatorial Optimization in Civil and Structural Engineering, B. H. V. Topping and A. I. Khan, Eds., pp. 95-100, Civil-Comp, Edinburgh, UK, 1993.

[151] C. A. C. Coello, "Theoretical and numerical constrainthandling techniques used with evolutionary algorithms: a survey of the state of the art," Computer Methods in Applied Mechanics and Engineering, vol. 191, no. 11-12, pp. 1245-1287, 2002.

[152] T. Back and M. Schütz, "Evolutionary strategies for mixedinteger optimization of optical multilayer systems," in Proceedings of the 4th Annual Conference on Evolutionary Programming, R. . McDonnel, R. G. Reynolds, and D. B. Fogel, Eds., pp. 33-51, MIT Press, Cambridge, Mass, USA.

[153] S. Rajasekaran, V. S. Mohan, and O. Khamis, "The optimisation of space structures using evolution strategies with functional networks," Engineering with Computers, vol. 20, no. 1, pp. 75-87, 2004.

[154] S. Rajasekaran, "Optimal laminate sequence of non-prismatic thin-walled composite spatial members of generic section," Composite Structures, vol. 70, no. 2, pp. 200-211, 2005.

[155] G. Ebenau, J. Rottschäfer, and G. Thierauf, "An advanced evolutionary strategy with an adaptive penalty function for mixed-discrete structural optimisation," Advances in Engineering Software, vol. 36, no. 1, pp. 29-38, 2005.

[156] O. Hasançebi, "Discrete approaches in evolution strategies based optimum design of steel frames," Structural Engineering and Mechanics, vol. 26, no. 2, pp. 191-210, 2007.

[157] O. Hasançebi, "Optimization of truss bridges within a specified design domain using evolution strategies," Engineering Optimization, vol. 39, no. 6, pp. 737-756, 2007. 
[158] O. Hasançebi, "Adaptive evolution strategies in structural optimization: Enhancing their computational performance with applications to large-scale structures," Computers and Structures, vol. 86, no. 1-2, pp. 119-132, 2008.

[159] V. Černý, "Thermodynamical approach to the traveling salesman problem: An efficient simulation algorithm," Journal of Optimization Theory and Applications, vol. 45, no. 1, pp. 41-51, 1985.

[160] W. A. Bennage and A. K. Dhingra, "Single and multiobjective structural optimization in discrete-continuous variables using simulated annealing," International Journal for Numerical Methods in Engineering, vol. 38, no. 16, pp. 2753-2773, 1995.

[161] N. Metropolis, A. W. Rosenbluth, M. N. Rosenbluth, A. H. Teller, and E. Teller, "Equation of state calculations by fast computing machines," The Journal of Chemical Physics, vol. 21, no. 6, pp. 1087-1092, 1953.

[162] R. J. Balling, "Optimal steel frame design by simulated annealing," Journal of Structural Engineering, vol. 117, no. 6, pp. 1780$1795,1991$.

[163] S. A. May and R. J. Balling, "A filtered simulated annealing strategy for discrete optimization of 3D steel frameworks," Structural Optimization, vol. 4, no. 3-4, pp. 142-148, 1992.

[164] R. K. Kincaid, "Minimizing distortion and internal forces in truss structures by simulated annealing," in Proceedings of 31st AIAA/ASME/ASCE/AHS/ASC Structural Materials and Dynamics Conference, pp. 327-333, Long Beach, Calif, USA, April 1990.

[165] R. K. Kincaid, "Minimizing distortion and internal forces in truss structures by simulated annealing," in Proceedings of 32nd AIAA/ASME/ASCE/AHS/ASC Structural Materials and Dynamics Conference, pp. 327-333, Baltimore, Md, USA, April 1990.

[166] G. S. Chen, R. J. Bruno, and M. Salama, "Optimal placement of active/passive members in truss structures using simulated annealing," AIAA Journal, vol. 29, no. 8, pp. 1327-1334, 1991.

[167] B. H. V. Topping, A. I. Khan, and J. P. B. Leite, “Topological design of truss structures using simulated annealing," in Neural Networks and Combinatorial Optimization in Civil and Structural Engineering, B. H. V. Topping and A. I. Khan, Eds., pp. 151-165, Civil-Comp Press, UK, 1993.

[168] J. P. B. Leite and B. H. V. Topping, "Parallel simulated annealing for structural optimization," Computers and Structures, vol. 73, no. 1-5, pp. 545-564, 1999.

[169] S. R. Tzan and C. P. Pantelides, "Annealing strategy for optimal structural design," Journal of Structural Engineering, vol. 122, no. 7, pp. 815-827, 1996.

[170] O. Hasançebi and F. Erbatur, "On efficient use of simulated annealing in complex structural optimization problems," Acta Mechanica, vol. 157, no. 1-4, pp. 27-50, 2002.

[171] O. Hasançebi and F. Erbatur, "Layout optimisation of trusses using simulated annealing," Advances in Engineering Software, vol. 33, no. 7-10, pp. 681-696, 2002.

[172] S. O. Degertekin, M. S. Hayalioglu, and M. Ulker, "A hybrid tabu-simulated annealing heuristic algorithm for optimum design of steel frames," Steel and Composite Structures, vol. 8, no. 6, pp. 475-490, 2008.

[173] O. Hasancebi, S. Carbas, and M. P. Saka, "Improving the performance of simulated annealing in structural optimization," Structural and Multidisciplinary Optimization, vol. 41, no. 2, pp. 189-203, 2010.
[174] O. Hasançebi and E. Doǧan, "Evaluation of topological forms for weight-effective optimum design of single-span steel truss bridges," Asian Journal of Civil Engineering, vol. 12, no. 4, pp. 431-448, 2011.

[175] J. Kennedy and R. Eberhart, "Particle swarm optimization," in Proceedings of the IEEE International Conference on Neural Networks, vol. 4, pp. 1942-1948, December 1995.

[176] J. Kennedy and R. Eberhart, Swarm Intellegence, Morgan Kaufman, 2001.

[177] G. Venter and J. Sobieszczanski-Sobieski, "Multidisciplinary optimization of a transport aircraft wing using particle swarm optimization," Structural and Multidisciplinary Optimization, vol. 26, no. 1-2, pp. 121-131, 2004.

[178] P. C. Fourie and A. A. Groenwold, "The particle swarm optimization algorithm in size and shape optimization," Structural and Multidisciplinary Optimization, vol. 23, no. 4, pp. 259-267, 2002.

[179] R. E. Perez and K. Behdinan, "Particle swarm approach for structural design optimization," Computers and Structures, vol. 85, no. 19-20, pp. 1579-1588, 2007.

[180] S. He, E. Prempain, and Q. H. Wu, "An improved particle swarm optimizer for mechanical design optimization problems," Engineering Optimization, vol. 36, no. 5, pp. 585-605, 2004.

[181] S. He, Q. H. Wu, J. Y. Wen, J. R. Saunders, and R. C. Paton, "A particle swarm optimizer with passive congregation," BioSystems, vol. 78, no. 1-3, pp. 135-147, 2004.

[182] L. J. Li, Z. B. Huang, F. Liu, and Q. H. Wu, "A heuristic particle swarm optimizer for optimization of pin connected structures," Computers and Structures, vol. 85, no. 7-8, pp. 340-349, 2007.

[183] E. Doğan and M. P. Saka, "Optimum design of unbraced steel frames to LRFD-AISC using particle swarm optimization," Advances in Engineering Software, vol. 46, pp. 27-34, 2012.

[184] A. Colorni, M. Dorigo, and V. Maniezzo, "Distributed optimization by ant colony," in Proceedings of 1st European Conference on Artificial Life, pp. 134-142, USA, 1991.

[185] M. Dorigo, Optimization, learning and natural algorithms [Ph.D. thesis], Dipartimento Elettronica e Informazione, Politecnico di Milano, Italy, 1992.

[186] M. Dorigo and T. Stützle, Ant Colony Optimization, A Bradford Book, MIT, USA, 2004.

[187] C. V. Camp and B. J. Bichon, "Design of space trusses using ant colony optimization," Journal of Structural Engineering, vol. 130, no. 5, pp. 741-751, 2004.

[188] C. V. Camp, B. J. Bichon, and S. P. Stovall, "Design of steel frames using ant colony optimization," Journal of Structural Engineering, vol. 131, no. 3, pp. 369-379, 2005.

[189] A. Kaveh and S. Shojaee, "Optimal design of skeletal structures using ant colony optimization," International Journal for Numerical Methods in Engineering, vol. 70, no. 5, pp. 563-581, 2007.

[190] J. A. Bland, "Automatic optimal design of structures using swarm intelligence," in Proceedings of the Annual Conference of the Canadian Society for Civil Engineering, pp. 1945-1954, Canada, June 2008.

[191] A. Kaveh and S. Talatahari, "An improved ant colony optimization for the design of planar steel frames," Engineering Structures, vol. 32, no. 3, pp. 864-873, 2010.

[192] W. Wang, S. Guo, and W. Yang, "Simultaneous partial topology and size optimization of a wing structure using ant colony and gradient based methods," Engineering Optimization, vol. 43, no. 4, pp. 433-446, 2011. 
[193] İ. Aydoğdu and M. P. Saka, "Ant colony optimization of irregular steel frames including elemental warping effect," Advances in Engineering Software, vol. 44, no. 1, pp. 150-169, 2012.

[194] Z. W. Geem, J. H. Kim, and G. V. Loganathan, "A new heuristic optimization algorithm: harmony search," Simulation, vol. 76, no. 2, pp. 60-68, 2001.

[195] Z. W. Geem, J. H. Kim, and G. V. Loganathan, "Harmony search optimization: application to pipe network design," International Journal of Modelling and Simulation, vol. 22, no. 2, pp. 125-133, 2002.

[196] K. S. Lee and Z. W. Geem, "A new structural optimization method based on the harmony search algorithm," Computers and Structures, vol. 82, no. 9-10, pp. 781-798, 2004.

[197] K. S. Lee and Z. W. Geem, "A new meta-heuristic algorithm for continuous engineering optimization: harmony search theory and practice," Computer Methods in Applied Mechanics and Engineering, vol. 194, no. 36-38, pp. 3902-3933, 2005.

[198] Z. W. Geem, K. S. Lee, and C. L. Tseng, "Harmony search for structural design," in Proceedings of the Genetic and Evolutionary Computation Conference (GECCO '05), pp. 651-652, Washington, DC, USA, June 2005.

[199] Z. W. Geem, "Optimal cost design of water distribution networks using harmony search," Engineering Optimization, vol. 38, no. 3, pp. 259-280, 2006.

[200] Z. W. Geem, "Novel derivative of harmony search algorithm for discrete design variables," Applied Mathematics and Computation, vol. 199, no. 1, pp. 223-230, 2008.

[201] Z. W. Geem, Ed., Music-Inspired Harmony Search Algorithm, Springer, 2009.

[202] Z. W. Geem, "Particle-swarm harmony search for water network design," Engineering Optimization, vol. 41, no. 4, pp. 297311, 2009.

[203] Z. W. Geem, Ed., Recent Advances in Harmony Search Algorithm, Springer, 2010.

[204] Z. W. Geem, Ed., Harmony Search Algorithms for Structural Design Optimization, Springer, 2010.

[205] Z. W. Geem, "Harmony search algorithm," 2011, http://www .harmonysearch.info/.

[206] Z. W. Geem and W. E. Roper, "Various continuous harmony search algorithms for web-based hydrologic parameter optimization," International Journal of Mathematical Modelling and Numerical Optimisation, vol. 1, no. 3, pp. 231-226, 2010.

[207] M. P. Saka, "Optimum geometry design of geodesic domes using harmony search algorithm," Advances in Structural Engineering, vol. 10, no. 6, pp. 595-606, 2007.

[208] S. Carbas and M. P. Saka, "A harmony search algorithm for optimum topology design of single layer lamella domes," in Proceedings of The 9th International Conference on Computational Structures Technology, B. H. V. Topping and M. Papadrakakis, Eds., no. 50, Civil-Comp Press, Scotland, UK, 2008.

[209] S. Çarbaş and M. P. Saka, "Optimum design of single layer network domes using harmony search method," Asian Journal of Civil Engineering, vol. 10, no. 1, pp. 97-112, 2009.

[210] S. Çarbaş and M. P. Saka, "Optimum topology design of various geometrically nonlinear latticed domes using improved harmony search method," Structural and Multidisciplinary Optimization, vol. 45, no. 3, pp. 377-399, 2011.

[211] M. P. Saka and F. Erdal, "Harmony search based algorithm for the optimum design of grillage systems to LRFD-AISC," Structural and Multidisciplinary Optimization, vol. 38, no. 1, pp. 25-41, 2009.
[212] M. P. Saka, "Optimum design of steel sway frames to BS5950 using harmony search algorithm," Journal of Constructional Steel Research, vol. 65, no. 1, pp. 36-43, 2009.

[213] S. O. Degertekin, "Optimum design of steel frames via harmony search algorithm," Studies in Computational Intelligence, vol. 239, pp. 51-78, 2009.

[214] S. O. Degertekin, M. S. Hayalioglu, and H. Gorgun, "Optimum design of geometrically non-linear steel frames with semirigid connections using a harmony search algorithm," Steel and Composite Structures, vol. 9, no. 6, pp. 535-555, 2009.

[215] M. P. Saka and O. Hasancebi, "Adaptive harmony search algorithm for design code optimization of steel structures," in Harmony Search Algorithms for Structural Design Optimization, Z. W. Geem, Ed., chapter 3, SCI 239, pp. 79-120, Springer, Berlin, Germany, 2009.

[216] O. Hasançebi, F. Erdal, and M. P. Saka, "An adaptive harmony search method for structural optimization," Journal of Structural Engineering, vol. 136, no. 4, pp. 419-431, 2010.

[217] F. Erdal, E. Doan, and M. P. Saka, "Optimum design of cellular beams using harmony search and particle swarm optimizers," Journal of Constructional Steel Research, vol. 67, no. 2, pp. 237247, 2011.

[218] M. P. Saka, I. Aydogdu, O. Hasancebi, and Z. W. Geem, "Harmony search algorithms in structural engineering," in Computational Optimization and Applications in Engineering and Industry, X.-S. Yang and S. Koziel, Eds., Chapter 6, Springer, Berlin, Germany, 2011.

[219] M. Mahdavi, M. Fesanghary, and E. Damangir, "An improved harmony search algorithm for solving optimization problems," Applied Mathematics and Computation, vol. 188, no. 2, pp. 15671579, 2007.

[220] M. G. H. Omran and M. Mahdavi, "Global-best harmony search," Applied Mathematics and Computation, vol. 198, no. 2, pp. 643-656, 2008.

[221] L. D. Santos Coelho and D. L. de Andrade Bernert, "An improved harmony search algorithm for synchronization of discrete-time chaotic systems," Chaos, Solitons and Fractals, vol. 41, no. 5, pp. 2526-2532, 2009.

[222] O. K. Erol and I. Eksin, "A new optimization method: Big BangBig Crunch," Advances in Engineering Software, vol. 37, no. 2, pp. 106-111, 2006.

[223] C. V. Camp, "Design of space trusses using big bang-big crunch optimization," Journal of Structural Engineering, vol. 133, no. 7, pp. 999-1008, 2007.

[224] A. Kaveh and S. Talatahari, "Size optimization of space trusses using Big Bang-Big Crunch algorithm," Computers and Structures, vol. 87, no. 17-18, pp. 1129-1140, 2009.

[225] A. Kaveh and S. Talatahari, "Optimal design of Schwedler and ribbed domes via hybrid Big Bang-Big Crunch algorithm," Journal of Constructional Steel Research, vol. 66, no. 3, pp. 412419, 2010.

[226] A. Kaveh and H. Abbasgholiha, "Optimum design of steel sway frames using Big Bang-Big Crunch algorithm," Asian Journal of Civil Engineering, vol. 12, no. 3, pp. 293-317, 2011.

[227] A. Kaveh and S. Talatahari, "A discrete particle swarm ant colony optimization for design of steel frames," Asian Journal of Civil Engineering, vol. 9, no. 6, pp. 563-575, 2008.

[228] A. Kaveh and S. Talatahari, "A particle swarm ant colony optimization for truss structures with discrete variables," Journal of Constructional Steel Research, vol. 65, no. 8-9, pp. 1558-1568, 2009. 
[229] A. Kaveh and S. Talatahari, "Hybrid algorithm of harmony search, particle swarm and ant colony for structural design optimization," Studies in Computational Intelligence, vol. 239, pp. 159-198, 2009.

[230] A. Kaveh and S. Talatahari, "Particle swarm optimizer, ant colony strategy and harmony search scheme hybridized for optimization of truss structures," Computers and Structures, vol. 87, no. 5-6, pp. 267-283, 2009.

[231] A. Kaveh, S. Talatahari, and B. Farahmand Azar, "An improved hpsaco for engineering optimum design problems," Asian Journal of Civil Engineering, vol. 12, no. 2, pp. 133-141, 2010.

[232] A. Kaveh and S. M. Rad, "Hybrid genetic algorithm and particle swarm optimization for the force method-based simultaneous analysis and design," Iranian Journal of Science and Technology, Transaction B, vol. 34, no. 1, pp. 15-34, 2010.

[233] A. Kaveh and S. Talatahari, "Optimum design of skeletal structures using imperialist competitive algorithm," Computers and Structures, vol. 88, no. 21-22, pp. 1220-1229, 2010.

[234] A. Kaveh and S. Talatahari, "A novel heuristic optimization method: charged system search," Acta Mechanica, vol. 213, pp. 267-289, 2010.

[235] A. Kaveh and S. Talatahari, "Optimal design of skeletal structures via the charged system search algorithm," Structural and Multidisciplinary Optimization, vol. 41, no. 6, pp. 893-911, 2010.

[236] A. Kaveh and S. Talatahari, "Charged system search for optimum grillage system design using the LRFD-AISC code," Journal of Constructional Steel Research, vol. 66, no. 6, pp. 767771, 2010.

[237] A. Kaveh and S. Talatahari, "A charged system search with a fly to boundary method for discrete optimum design of truss structures," Asian Journal of Civil Engineering, vol. 11, no. 3, pp. 277-293, 2010.

[238] A. Kaveh and S. Talatahari, "Geometry and topology optimization of geodesic domes using charged system search," Structural and Multidisciplinary Optimization, vol. 43, no. 2, pp. 215-229, 2011.

[239] A. Kaveh and A. Zolghadr, "Shape and size optimization of truss structures with frequency constraints using enhanced charged system search algorithm," Asian Journal of Civil Engineering, vol. 12, no. 4, pp. 487-509, 2011.

[240] A. Kaveh and S. Talatahari, "Charged system search for optimal design of frame structures," Applied Soft Computing, vol. 12, pp. 382-393, 2012.

[241] A. Kaveh and T. Bakhspoori, "Optimum design of steel frames using cuckoo search algorithm with levy flights," The Structural Design of Tall and Special Buildings. In press.

[242] X. -S Yang and S. Deb, "Engineering Optimization by Cuckoo Search," International Journal of Mathematical Modelling and Numerical Optimisation, vol. 1, no. 4, pp. 330-343, 2010.

[243] A. H. Gandomi, X.-S. Yang, and A. H. Alavi, "Cuckoo search algorithm: a metaheuristic approach to solve structural optimization problems," Engineering with Computers. In press.

[244] O. Hasançebi, S. Çarbaş, E. Doğan, F. Erdal, and M. P. Saka, "Performance evaluation of metaheuristic search techniques in the optimum design of real size pin jointed structures," Computers and Structures, vol. 87, no. 5-6, pp. 284-302, 2009.

[245] O. Hasançebi, S. Çarbaş, E. Doğan, F. Erdal, and M. P. Saka, "Comparison of non-deterministic search techniques in the optimum design of real size steel frames," Computers and Structures, vol. 88, no. 17-18, pp. 1033-1048, 2010.
[246] A. Kaveh and S. Talatahari, "Optimal design of single layer domes using meta-heuristic algorithms; a comparative study," International Journal of Space Structures, vol. 25, no. 4, pp. 217227,2010 


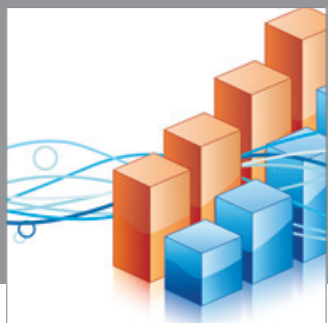

Advances in

Operations Research

mansans

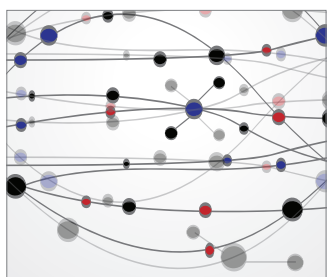

The Scientific World Journal
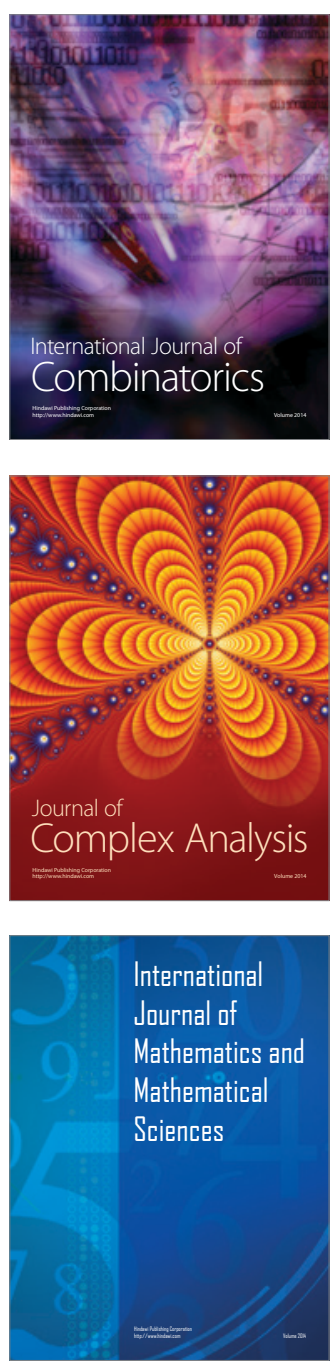
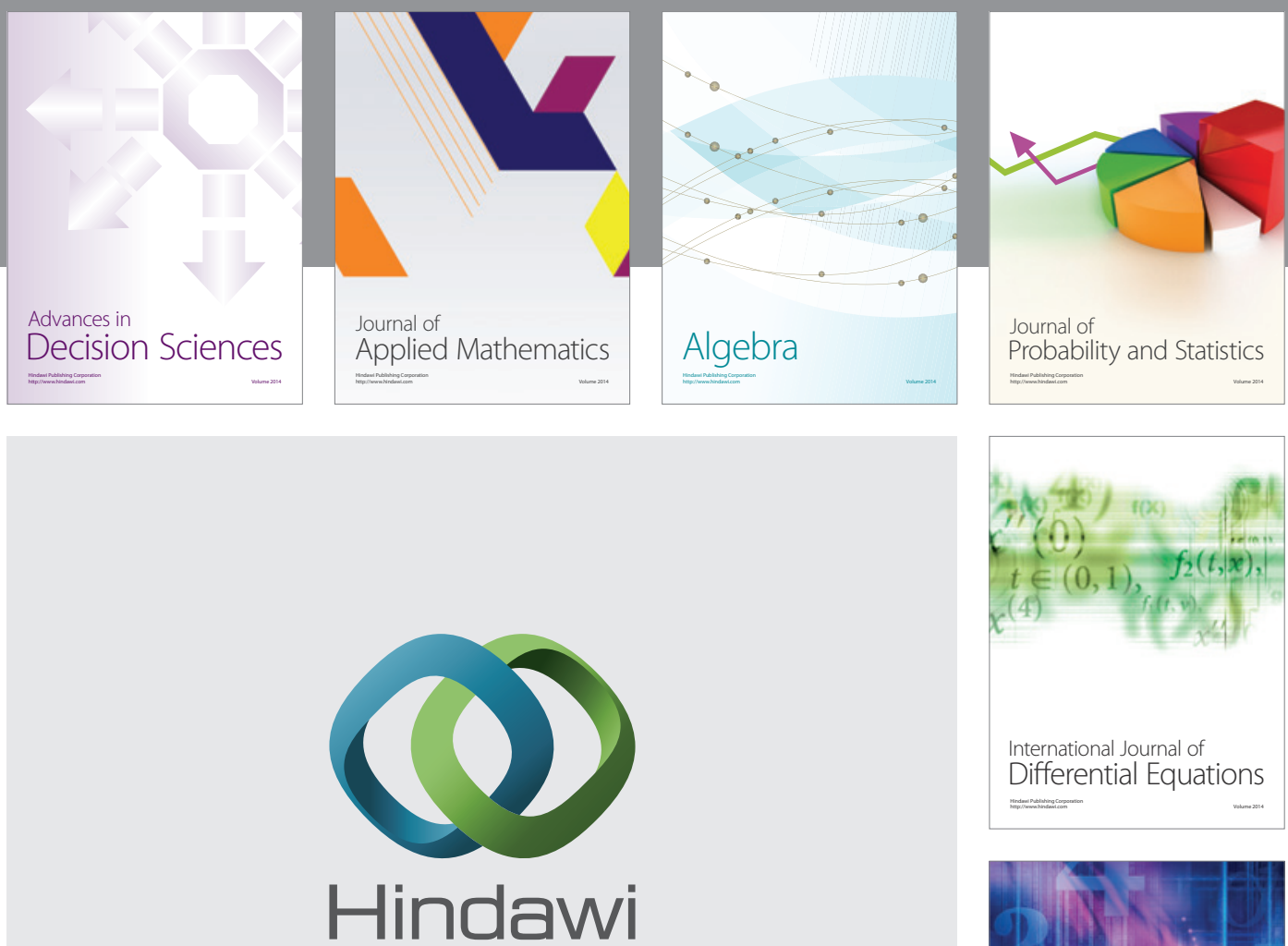

Submit your manuscripts at http://www.hindawi.com
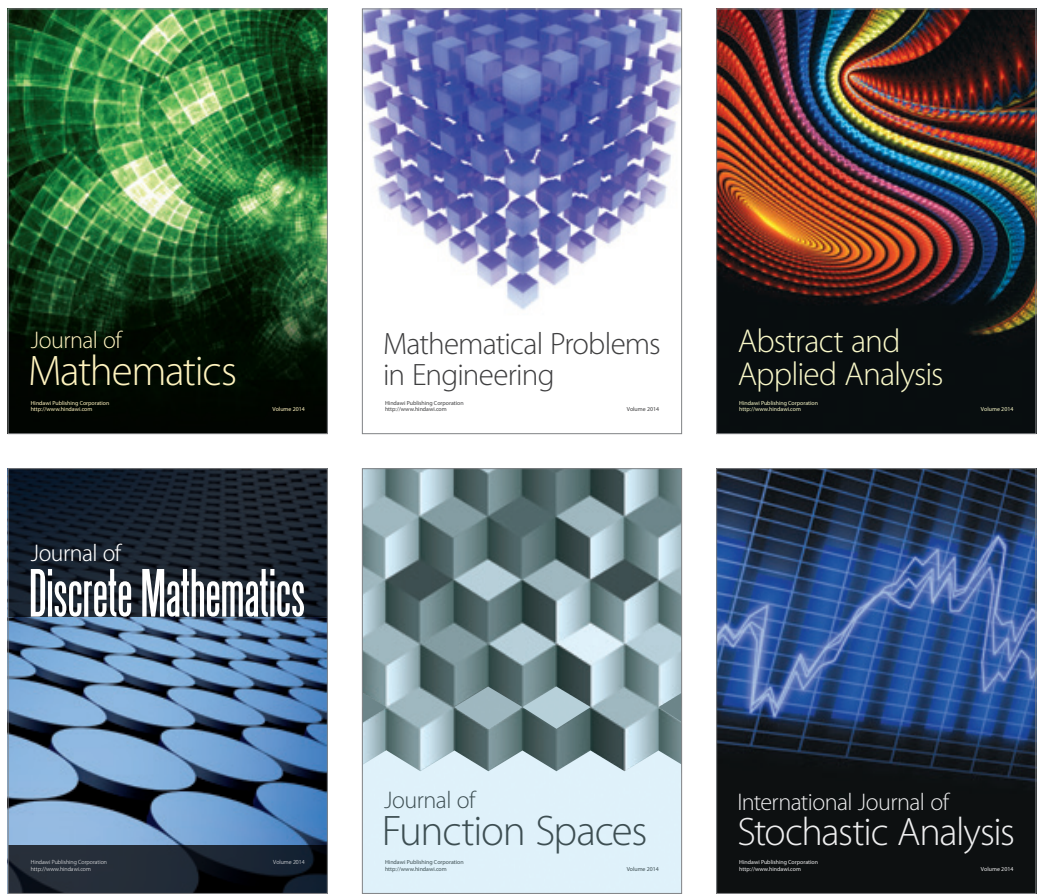

Journal of

Function Spaces

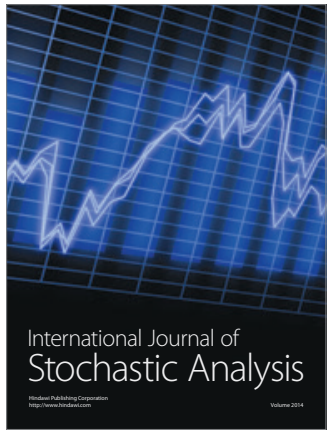

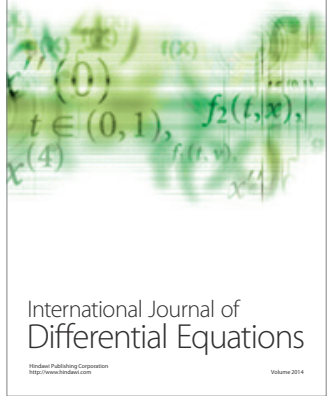
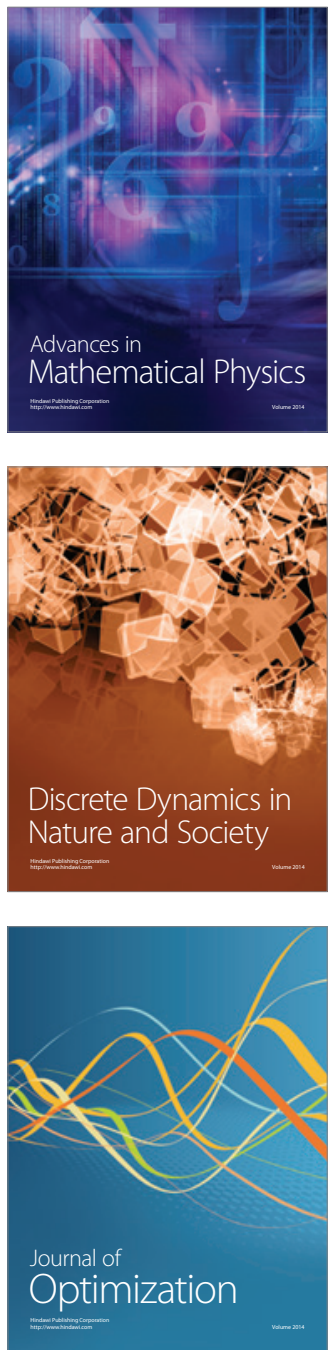